\title{
Aspekt, Temporalität und Argumentstruktur bei attributiven Partizipien des Deutschen*
}

\author{
ANJA LÜBBE \& IRENE RAPP
}

Abstract

German has two participles, both of which occur in prenominal position. Traditionally, both participles are considered to be associated with particular semantic and syntactic features. The present participle (malend 'painting') is subject-oriented; it has imperfective aspect and indicates simultaneity of the participle event and the matrix clause event. The past participle (gemalt 'painted') is object-oriented; it has perfective aspect and indicates that the participle event precedes the event of the matrix clause. In this paper, we will first argue that aspect is indeed an inherent - hence invariant - feature of the participle morphology: The present participle always presents an event as ongoing, whereas the past participle presents it as completed. Secondly, we will show that participles - as non-finite verb forms - lack tense. Hence, their temporal properties vary according to lexical, syntactic and pragmatic factors. It will be demonstrated that the unmarked temporal readings of the participles are due to the maxim of relation. Finally, we will argue that the argument selection properties of both participles are grammatically determined; however, these properties can be altered if certain conditions are met.

Keywords: participle, prenominal attribute, aspect, temporality, argument alternation

* Dieser Aufsatz ist im Rahmen des Projekts A5 Bedeutungskonstitution bei infiniten Verbalformen des von der DFG geförderten Sonderforschungsbereichs 833 Bedeutungskonstitution: Dynamik und Adaptivität sprachlicher Strukturen entstanden. Für Anregungen und Diskussionen bedanken wir uns bei Sebastian Bücking, Christian Fortmann, Ewald Lang und Alexander Pfaff; für die kritische Kommentierung einer Erstfassung bei Klaus von Heusinger und zwei Gutachtern der Zeitschrift für Sprachwissenschaft. Gewidmet ist der Aufsatz Brigitte Handwerker, die uns im Rahmen zweier Vorträge an der Humboldt-Universität eine Reihe von Denkanstößen gegeben hat. 


\section{1. Überblick}

In der Tradition von Gunnar Bech (1983) werden Partizipien als infinite Verbalformen betrachtet, die in adjektivtypischen Positionen - attributiv bzw. prädikativ - auftreten. In pränominaler Position kann im Deutschen sowohl das Partizip I als auch das Partizip II auftreten, allerdings mit unterschiedlichem Argumentbezug:

transitives Basisverb:

a. Die einen Hasen malende Frau pfiff.

b. Der gemalte Hase begeisterte die Kinder.

intransitives Basisverb:

a. Die arbeitende Frau seufzte.

b. *Die gearbeitete Frau war müde.

Das Partizip I bezieht sich auf das Subjekt des Basisverbs, vgl. (1a) und (2a), das Partizip II dagegen auf dessen direktes Objekt, vgl. (1b) ${ }^{1}-$ dies schließt eine pränominale Verwendung des Partizip II von Verben ohne direktes Objekt im Allgemeinen aus, vgl. (2b). Ausgenommen von dieser Restriktion sind Verben, die als Perfektauxiliar sein selegieren, wie z. B. einschlafen, ankommen, aufwachen. Diese Verben - im Folgenden als intransitive sein-Verben bezeichnet - erlauben eine pränominale Verwendung des Partizip II, obwohl sie kein direktes Objekt besitzen. Hier bezieht sich das Partizip II ebenso wie das Partizip I auf das Subjekt: ${ }^{2}$

1. Wir sprechen von Subjekt- bzw. Objektbezug, wenn das Bezugsnomen des Partizips dem Subjekt bzw. Akkusativobjekt des Verbs entspricht, das dem Partizip zugrunde liegt. Die Einstufung des Partizip I als subjektbezogen und des Partizip II als objektbezogen gilt auch für sekundäre Prädikationen:

(i) Lachend kam das Kind mir entgegen. $\rightarrow$ Das Kind lachte.

(ii) Geschlagen verließ der Spieler das Feld. $\rightarrow$ Jemand hatte den Spieler geschlagen.

Auf die Eigenarten dieser Konstruktionen kann hier jedoch nicht näher eingegangen werden.

2. Im generativen Paradigma werden Verben dieser Gruppe zumeist als unakkusative (oder ergative) Verben bezeichnet (vgl. Perlmutter 1978; Burzio 1981). Die Unakkusativitätshypothese besagt, dass das Subjekt unakkusativer Verben tiefenstrukturell Objektstatus hat; der Argumentbezug des pränominalen Partizip II könnte somit durch die Annahme erfasst werden, dass es in jedem Fall den Bezug auf ein tiefenstrukturelles Objekt herstellt. Neben Auxiliarselektion und attributiver Verwendbarkeit des Partizip II werden in der Literatur verschiedene weitere Diagnostiken für Unakkusativität aufgeführt; so z. B. dass Unakkusative kein Passiv sowie keine -er-Nominalisierungen erlauben (vgl. für das Deutsche Grewendorf 1989). Diese Diagnostiken liefern jedoch keineswegs einheitliche Ergebnisse, so dass sowohl die Einschlägigkeit jeder einzelnen Diagnostik umstritten ist als auch die Annahme, dass "Unakkusative" überhaupt eine morpho-syntaktisch einheitliche Gruppe bilden (vgl. Strobel 2008). Wir werden daher in diesem Aufsatz nicht von der Unakkusativitätshypothese ausgehen; wir begnügen uns mit der Feststellung, dass im unmarkierten Fall genau diejenigen intransitiven Ver- 
a. Die einschlafende Frau stöhnte.

b. Die eingeschlafene Frau lag auf dem Boden.

Die germanistische Forschung der letzten Jahrzehnte hat vorwiegend den kategorialen Status der Partizipien ("Mittelwort", Verbform oder Adjektiv?) untersucht. Im Mittelpunkt standen dabei zumeist ihre morphologischen und syntaktischen Eigenschaften (Lenz 1993; Fuhrhop \& Teuber 2000 und die Beiträge in Bresson \& Dalmas 1994). Für das sogenannte Zustandspassiv fanden sich zunehmend Argumente für eine Analyse als Kopulakonstruktion, bei der das Partizip II als Adjektiv eingestuft wird (Rapp 1996, 1997; Maienborn 2007). Eine derartige Klassifikation scheint zunächst auch für pränominale Partizipien plausibel - vor allem auf Grund der hier auftretenden adjektivischen Flexion (vgl. Marillier 1994; Valentin 1994; Quintin 1994). Allerdings zeigt eine Reihe von Phänomenen, dass bei pränominalen Partizipien die ursprünglich verbalen Eigenschaften in weit stärkerem Maße erhalten sind als beim Zustandspassiv (vgl. Faucher 1994; Poitou 1994). So sind beim pränominalen Partizip I alle Argumente des Basisverbs realisierbar, wobei ihr Status als fakultativ bzw. obligatorisch erhalten bleibt (vgl. $4 \mathrm{a}-4 \mathrm{~d}$, siehe auch Toman 1986). Zudem ist das Verbalereignis immer für adverbiale Modifikation zugänglich (4e):
a. Die Frau liest ein Buch. / Die ein Buch lesende Frau pfiff.
b. Die Frau liest. / Die lesende Frau pfiff.
c. Die Laterne beleuchtete die Straße. I Die die Straße beleuch- tende Laterne fiel um.
d. *Die Laterne beleuchtete. I *Die beleuchtende Laterne fiel um.
e. Die eifrig lesende Frau pfiff.

Auch beim pränominalen Partizip II ist adverbiale Modifikation stets möglich (5a), außerdem kann das Subjekt transitiver Verben als fakultative von-Phrase auftreten (5b). Das Zustandspassiv erlaubt dagegen im Allgemeinen weder adverbiale Modifikation noch agentive von-Phrasen $(6 a, b):^{3}$
a. Peter erblickte die am vorigen Tag zerstörte Stadt.
b. Peter erblickte die von den Feinden zerstörte Stadt.
a. *Die Stadt ist am vorigen Tag zerstört.
b. *Die Stadt ist von den Feinden zerstört.

ben, die ihr Perfekt mit sein bilden, die pränominale Verwendung des Partizip II mit

Subjektbezug zulassen. Abweichende Fälle werden in Abschnitt 3 behandelt.

3. Zu einer genaueren Darstellung der Zustandspassivrestriktionen s. Abschnitt 2.1.2. 
Wir werden daher im Folgenden davon ausgehen, dass pränominale Partizipien in syntakto-semantischer Hinsicht die verbalen Eigenschaften ihrer Basis beibehalten können (vgl. Rapp 1997, 2000). ${ }^{4}$

Nun ist augenfällig, dass sich die beiden Partizipien nicht nur bzgl. der Argumentrealisierung, sondern auch hinsichtlich ihrer aspektuellen und temporalen Interpretation grundlegend unterscheiden. Während beim Partizip I lesend das Verbalereignis als im Verlauf befindlich angesehen wird (imperfektiver Aspekt), bezeichnet das Partizip II zerstört ein abgeschlossenes Ereignis (perfektiver Aspekt). Dem Aspekt des attributiven Partizips entspricht eine (jeweils unterschiedliche) temporale Relation zum finiten Verb des Satzes: Wir verstehen (1a) so, dass die Frau beim Malen pfiff - das Partizipialereignis (malen) und das Matrixereignis (pfeifen) sind also gleichzeitig. ${ }^{5}$ In (1b) dagegen begeisterte die Kinder ein Hase, der bereits gemalt wurde - hier ist das Partizipialereignis ( $m a-$ len) vorzeitig zum Matrixereignis (begeistern). Im Falle von intransitiven sein-Verben wie einschlafen, ankommen, aufwachen, bei denen pränominale Partizipien stets Subjektbezug aufweisen, reduziert sich der Unterschied zwischen Partizip I und Partizip II auf deren aspektuelle (und temporale) Eigenschaften (vgl. Weber 2000). Es ergibt sich folgendes Schema:

Tabelle 1: Argumentrealisierung und aspektuell-temporale Eigenschaften der Partizipien

\begin{tabular}{|c|c|c|}
\hline & Subjektbezug & Objektbezug \\
\hline imperfektiv-gleichzeitig & $\begin{array}{l}\text { PI: die lesende Frau } \\
\text { das einschlafende Kind }\end{array}$ & - \\
\hline perfektiv-vorzeitig & PII: das eingeschlafene Kind & PII: die zerstörte Stadt \\
\hline
\end{tabular}

In diesem Aufsatz soll es um interpretatorische Flexibilität der Partizipien bezüglich der genannten aspektuellen, temporalen und argumentstrukturellen Eigenschaften gehen. Anders ausgedrückt: Wir werden untersuchen, wie fest die Verknüpfung dieser syntakto-semantischen Eigenschaften mit den beiden Partizipialformen ist. Unsere zentrale These lautet, dass Partizipien durch ihre morphologischen Merkmale auf einen

4. Die morphologische Struktur der Partizipien ist nicht Thema dieses Aufsatzes. Toman (1986) nimmt an, dass sich beim Partizip I -end mit einem Verbalstamm verbindet; wir folgen dagegen Wunderlich (1987) und Zifonun et al. (1997: 2206) darin, dass das Partizipialsuffix $-d$ an den verbalen Infinitiv tritt: Die Verbindung von /nd/ mit dem Verbstamm ist aus phonologischen Gründen unplausibel, da in diesem Falle nach den Regeln der Schwa-Epenthese (vgl. Wiese 1986) eine Realisierung als [nəd] zu erwarten wäre.

5. Von der ebenfalls möglichen habituellen Lesart - malen z. B. als Hobby der Frau soll in diesem Aufsatz abgesehen werden. 
bestimmten Aspekt festgelegt sind; dieser kann in keinem Falle verändert werden (vgl. Ehrich \& Vater 1989 und Weber 2000). Die temporalen Eigenschaften attributiver Partizipien sind dagegen nicht morphologisch determiniert, sondern ergeben sich entweder durch lexikalische Mittel (Verwendung von Zeitadverbialen) oder auf pragmatischer Ebene als Implikaturen. Aspekt und temporale Eigenschaften attributiver Partizipien werden in Abschnitt 2 dargestellt; in 2.1. werden die den Partizipien inhärierenden aspektuellen Merkmale aufgezeigt, in 2.2. gehen wir auf die verschiedenen Arten temporaler Bestimmung ein. ${ }^{6}$

Der Argumentbezug wiederum ist auf grammatischer Ebene festgelegt. Anders als beim Aspekt treten hier jedoch nicht selten Abweichungen auf:
a. das stattgefundene
- Das Treffen hat stattgefunden. Treffen
b. der gehuldigte König - Man hat dem König gehuldigt.

Im Falle von stattgefunden besteht Subjektbezug des Partizip II, obwohl das Basisverb haben als Perfektauxiliar selegiert. Im Falle von gehuldigt bezieht sich das Partizip II auf ein Dativobjekt. Diese und andere Formen des abweichenden Gebrauchs hinsichtlich des Argumentbezugs finden sich bereits in älteren Grammatiken (Paul 1920: Kapitel 7, $\$ \$ 317$, 326 und Behaghel 1924: §758) erwähnt, sie wurden und werden jedoch zumeist ohne weitere Erklärungen als inakzeptabel eingestuft. U.E. bestehen hier zwar vordergründig Regelverstöße, bei näherer Betrachtung zeigt sich aber, dass sie durchaus ihre eigene Berechtigung und ihre internen Regularitäten haben. Wir werden in Abschnitt 3 untersuchen, unter welchen Bedingungen Partizipien mit abweichendem Argumentbezug verwendet werden können. Vorweg ist zu betonen, dass diese Adaption einen ganz anderen Stellenwert hat als die bereits erwähnte temporale Adaption: während es sich bei letzterer um eine grammatisch reguläre Bedeutungsanreicherung handelt, wird bei der Argumentadaption eine grammatisch irreguläre Struktur unter gewissen Bedingungen akzeptiert.

Um unsere Auffassungen empirisch zu stützen, haben wir für illustrierende Beispielsätze auf Korpusdaten zurückgegriffen, insbesondere, um das Vorkommen von markierten temporalen Bedeutungsanreicherungen und von Argumentadaptionen zu belegen. ${ }^{7}$

6. Partizipien in prädikativen Konstruktionen verhalten sich bzgl. der temporalen Interpretation sehr viel restriktiver als attributive Partizipien, worauf wir hier jedoch nicht eingehen.

7. Korpusbelege sind von introspektiven Beispielen durch die andersartige Nummerierung (1-1, 1-2 vs. 1a, 1b) abgehoben. Sämtliche Belege stammen, wenn nicht anders vermerkt, aus den Korpora des DWDS der Berlin-Brandenburgischen Akademie der Wissenschaften (www.dwds.de), Korpus: DIE ZEIT/1980-2010. 


\section{Aspekt und Temporalität}

Im Einklang mit Reichenbach (1947), Bäuerle (1978) und Klein (1994) unterscheiden wir drei Zeitparameter: die Äußerungszeit, die Ereigniszeit und die Topikzeit. Unter Ereigniszeit verstehen wir die gesamte Zeitspanne, zu der ein Ereignis ${ }^{8}$ gegeben ist. Die Topikzeit ist dagegen die Zeitspanne, die der Sprecher bei seiner Äußerung fokussiert. Klein (1994) verdeutlicht den Unterschied zwischen Ereigniszeit und Topikzeit anhand einer Zeugenbefragung vor Gericht:

Judge: What did you notice when you entered the room?

Witness: A man was lying on the floor. He was Chinese or Japanese.

(Klein 1994: 40)

Die Topikzeit der Antwort ist hier kontextuell durch die explizite Frage des Richters gegeben: es geht genau um die Zeitspanne, zu der der Zeuge das Zimmer betreten hat. Nun kann der Mann durchaus schon vor oder nach der recht engen Topikzeit auf dem Boden liegen. Die Ereigniszeit differiert also offenkundig von der Topikzeit. Wir veranschaulichen dies im Folgenden in Anlehnung an Klein (1994) durch Zeitschemata, in denen die Ereigniszeit durch waagerechte Striche und die Topikzeit durch eckige Klammern gekennzeichnet ist:

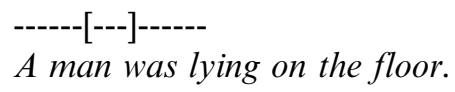

(Klein 1994: 41)

Die Relation zwischen Ereigniszeit und Topikzeit definiert Klein (1994) als Aspekt. Bei einer Inklusion der Topikzeit in der Ereigniszeit liegt imperfektiver Aspekt vor, was im Englischen durch den Progressiv markiert wird. Nicht nötig ist diese explizite Aspektmarkierung bei permanenten Zuständen (wie Chinese-Sein), die in jedem Falle als imperfektiv bzgl. der Topikzeit aufgefasst werden. Aufschlussreich ist die Beobachtung, dass auch bei der Darstellung permanenter Zustände die jeweilige Erzählzeit (hier: Präteritum) beibehalten und nicht etwa ein Präsens verwendet wird. Dies macht deutlich, dass Tempus tatsächlich die Relation zwischen Äußerungszeit und Topikzeit markiert und nicht etwa unmittelbar die Relation zwischen Äußerungszeit und Ereigniszeit.

Im Folgenden werden wir die Klein'sche Begrifflichkeit für die Zwecke unserer Untersuchung präzisieren. Die Begriffe Tempus und Aspekt reservieren wir für Fälle, bei denen die jeweiligen Relationen durch gram-

8. Für die Zwecke dieses Aufsatzes sind unter dem Begriff Ereignis alle Aktionsarten (also auch Zustände) zusammengefasst. 
matische Mittel ausgedrückt werden. In allen anderen Fällen sprechen wir von Temporalität und Aspektualität.

Tempus und Temporalität betreffen die Situierung der Topikzeit. Als Tempus (im engeren Sinne) bezeichnen wir die durch die finite Flexion grammatisch fixierte Beziehung zwischen Äußerungszeit und Topikzeit. Zu beachten ist dabei, dass durch das Tempus zwar eine Restriktion der Topikzeit erfolgt, jedoch keine exakte Festlegung. So gibt das Präteritum an, dass die Topikzeit vor der Äußerungszeit liegt, eine genauere Bestimmung muss jedoch durch pragmatische Faktoren erfolgen. Temporalität kann zudem durch Zeitadverbiale markiert werden. Im Falle von Punktadverbialen (wie um $17 \mathrm{Uhr}$ ) ist die Topikzeit ein sehr kleines Zeitintervall, das den betreffenden Zeitpunkt umgibt. Zeitraumsetzende Adverbiale wie heute, letztes Jahr benennen dagegen einen Zeitraum, innerhalb dessen die Topikzeit angesiedelt ist. Letzteres sei in Anlehnung an Bäuerle (1978) an einem Beispiel illustriert:

\section{Heute ging Peter in "Vom Winde verweht".}

Sowohl das Tempus (Präteritum) als auch das Zeitadverbiale (heute) restringieren die Topikzeit: als Topikzeitrahmen ergibt sich hier folglich der Teil von heute, der vor der Äußerungszeit liegt. Voraussetzung für die Verwendung von Zeitadverbialen ist, wie Bäuerle (1978) feststellt, dass die Adverbialrestriktion der Tempusrestriktion nicht widerspricht (*Morgen ging Peter in "Vom Winde verweht".). Eine Fixierung der Topikzeit erfolgt jedoch auch bei Verwendung eines zeitraumsetzenden Adverbiales auf der Basis pragmatischen Wissens: normalerweise wissen wir, dass das Kino vormittags noch nicht geöffnet hat, möglicherweise ist uns auch bekannt, dass der Beginn des Films um $17 \mathrm{Uhr}$ ist und ähnliches. Dies bedeutet, dass zeitraumsetzende Adverbiale lediglich eine maximale Topikzeit angeben, die kontextuell zumeist noch weiter restringiert wird.

Aspekt und Aspektualität betreffen das Verhältnis von Topikzeit und Ereigniszeit. Unter Aspekt verstehen wir wiederum den Ausdruck von Aspektualität durch spezifische grammatische Mittel (wie z. B. den englischen Progressiv als Imperfektivmarker). Perfektiver Aspekt liegt dann vor, wenn die Ereigniszeit innerhalb der Topikzeit liegt; d. h. das Geschehen wird als von außen Umgrenztes angesehen (Außenperspektive). Imperfektiver Aspekt liegt vor, wenn die Topikzeit innerhalb der Ereigniszeit liegt, d.h. innerhalb des sich im Verlauf befindenden Geschehens angesiedelt ist (Innenperspektive). ${ }^{9}$ Für das Deutsche gehen wir mit

9. Zur Terminologie vgl. Zifonun et al. (1997: 1861). 
Klein (1994) davon aus, dass finite Verben Tempus, jedoch nicht Aspekt markieren. Einzige Interpretationsbedingung ist, dass Ereigniszeit und Topikzeit überlappen. Betrachten wir hierzu zwei Beispiele, bei denen die Topikzeit durch ein Punktadverbiale festgelegt ist:

\section{a. Um 12 Uhr strich Paul sein Häuschen an. \\ b. Um 12 Uhr warf Paul den Stuhl um.}

Als Topikzeit ergibt sich in beiden Fällen ein minimal kleines Zeitintervall, das den Zeitpunkt um $12 \mathrm{Uhr}$ umgibt. Ob eine Inklusionsbeziehung zwischen Ereigniszeit und Topikzeit besteht, ist nicht grammatisch vorgegeben. Vielmehr wird die Beziehung zwischen den beiden Zeiten durch verschiedene Faktoren bestimmt. Neben pragmatischen Gesichtspunkten spielt v. a. die semantische Verbklasse eine wichtige Rolle: so legt das durative Verb anstreichen in (11a) nahe, dass die Topikzeit innerhalb der Ereigniszeit liegt, das punktuelle Verb umwerfen in (11b) dagegen macht eine Inklusion der Ereigniszeit in der Topikzeit (bzw. die Identität beider Zeiten) wahrscheinlich. Auch bei zeitraumsetzenden Adverbialen sehen wir, dass Aspekt nicht grammatisch vorgegeben ist:
a. Gestern strich Paul sein Häuschen an.
b. Gestern warf Paul den Stuhl um.

In (12a) kann die Ereigniszeit über die maximale Topikzeit gestern hinausgehen, da ein duratives Verb vorliegt (und da wir wissen, dass der Prozess des Häuschen-Anstreichens durchaus mehrere Tage in Anspruch nehmen kann). Eine derartige Interpretation ist beim punktuellen Verb umwerfen in (12b) dagegen nicht möglich.

Während also finite Verben Tempus, nicht aber Aspekt morphologisch kodieren, sind Partizipien - so unsere These - tempuslose, jedoch aspektmarkierte infinite Formen. Dies heißt einerseits, dass ihre Topikzeit nicht auf grammatische Weise, sondern durch Zeitadverbiale bzw. durch Rekurs auf die Pragmatik festgelegt ist. Andererseits bedeutet es, dass die Beziehung zwischen Ereigniszeit und Topikzeit durch die Partizipialmorphologie grammatisch determiniert ist: das Partizip I markiert stets den imperfektiven, das Partizip II den perfektiven Aspekt.

Um den Aspekt der Partizipien darstellen zu können, wählen wir zunächst Beispiele, bei denen die Topikzeit durch ein Adverbiale lexikalisch restringiert ist. Durch die Verwendung unterschiedlicher Zeitadverbiale und unterschiedlicher semantischer Verbklassen ergeben sich ganz verschiedenartige Konstellationen, die in 2.1. dargestellt werden. Es lässt sich hierbei belegen, dass die spezifische Aspektmarkierung der beiden Partizipien in allen betrachteten Fällen besteht. In 2.2. gehen wir auf die 
Temporalität von attributiven Partizipien ein. $\mathrm{Zu}$ untersuchen ist, auf welche Weise die Topikzeit gewählt wird, wenn kein Zeitadverbiale vorhanden ist. Wir werden zeigen, dass hierfür nicht nur Weltwissen, sondern in starkem Maße generalisierte konversationelle Implikaturen ausschlaggebend sind.

\subsection{Aspekt}

2.1.1. Aspekt beim attributiven Partizip I. Betrachten wir zunächst die Verbindung des Partizip I mit Punktadverbialen (um 12 Uhr). Die Topikzeit ist hier ein minimal kleines Intervall, nämlich die unmittelbare Umgebung von 12 Uhr. Im Falle des Partizip I wird diese Topikzeit von der Ereigniszeit umschlossen:
a. accomplishment:
b. activity:
der um 12 Uhr ein Bild malende Junge
c. state:
der um 12 Uhr arbeitende Junge
d. achievement: die um 12 Uhr im Bett liegende Studentin die um 12 Uhr ein Buch kaufende Frau I das um 12 Uhr einschlafende Kind

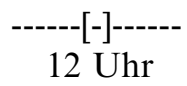

Alle Beispiele unter (13) werden so interpretiert, dass die Ereigniszeit um $12 \mathrm{Uhr}$ andauert; dies gilt sogar für ein als quasi-punktuell verstandenes achievement-Verb wie kaufen. Wir können Imperfektivität - wie auch viele Grammatiken dies tun - als Innenperspektive bezeichnen: das Ereignis ist zur betrachteten Zeit im Gange. ${ }^{10}$ Eine weniger eindeutige Lage ergibt sich bei der Kombination des Partizip I mit zeitraumsetzenden Adverbialen wie gestern oder letztes Jahr. Diese bestimmen die Topikzeit nicht genau, sie restringieren sie lediglich $-\mathrm{d}$.h. die Topikzeit liegt irgendwo innerhalb des Adverbialzeitraums:

\section{der gestern sein Häuschen anstreichende Mann}

Es ist klar, dass sich durch Beispiele wie (15) keine direkte Evidenz für die Imperfektivität des Partizip I finden lässt. Imperfektivität kann zwar

10. Im Falle telischer Verben ergeben sich durch die Imperfektivität diverse Komplikationen. So muss das Verbalereignis, das zur Topikzeit im Gange ist, nicht vollendet werden: (i) Der um 12 Uhr die Straße überquerende Politiker wurde von einem Auto erfasst. Offenbar ist das hier relevante Ereignis kein ganzes die-Straße-Überqueren, sondern lediglich eine Vorstufe dazu ist. U.E. sind die Überlegungen, die z. B. Landman (1992) für die englische Verlaufsform anstellt, auf die Imperfektivität beim Partizip I völlig übertragbar. 
bzgl. des Gesamtintervalls gestern bestehen (16). Ebenso ist es jedoch möglich, dass als Topikzeit ein Teil von gestern gewählt wird und nur dieses - sprachlich nicht genau benannte - Teilintervall innerhalb der Ereigniszeit liegt (17):

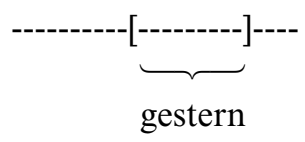

$$
\text { [-[---]- ] }
$$

Es ist von verschiedenen Faktoren abhängig, welche Interpretation wir wählen. Den größten Einfluss hat dabei - neben pragmatischen Gesichtspunkten - wiederum die lexikalische Semantik des Basisverbs. Accomplishments (wie anstreichen, schreiben, malen) erlauben aufgrund ihrer Durativität beide Lesarten, ebenso activities und states. Ein achievement-Verb wie kaufen ermöglicht dagegen im Normalfall nur Imperfektivität bzgl. eines quasi-punktuellen Teils des Gesamtintervalls:
a. accomplishment: der gestern sein Häuschen anstreichende Mann
b. activity: der zwischen drei und vier arbeitende Junge
c. state: die letztes Jahr in Tübingen wohnenden Studenten
d. achievement: der gestern ein Buch kaufende Mann I das gestern einschlafende Kind

Fassen wir zusammen: Punktadverbiale illustrieren die Imperfektivität des Partizip I in überzeugender Weise. Zeitraumsetzende Adverbiale sind dagegen beim Partizip I wenig aufschlussreich. Eine Inklusion der gesamten Adverbialzeit in der Ereigniszeit ist nicht notwendigerweise gegeben, da die Topikzeit hier nicht der gesamten Adverbialzeit entsprechen muss: Imperfektivität kann immer auch bzgl. eines sprachlich nicht manifesten Teilintervalls der Adverbialzeit bestehen. Signifikant wird die Betrachtung von Adverbialen wie gestern oder letztes Jahr erst durch den Vergleich des Partizip I mit dem Partizip II: der perfektive Charakter des Partizip II führt dazu, dass im Gegensatz zum Partizip I hier die Ereigniszeit tatsächlich innerhalb der Adverbialzeit liegen muss. 
2.1.2. Aspekt beim attributiven Partizip II. Die Untersuchung des Aspekts beim pränominalen Partizip II sieht sich mit einem Abgrenzungsproblem konfrontiert. Viele Verben erlauben eine Adjektivierung des Partizip II; in Rapp (1996) und Maienborn (2007) wurde gezeigt, dass das sogenannte Zustandspassiv immer als Kopulakonstruktion analysiert werden muss, bei der ein adjektiviertes Partizip II auftritt. Die pränominale Position ist liberaler. Natürlich lässt sie adjektivierte Partizipien zu; daneben sind - wie bereits gezeigt - hier aber auch verbale Partizipien möglich. Um den Aspekt des verbalen attributiven Partizip II zu untersuchen, muss also sichergestellt werden, dass wirklich ein verbales und kein adjektivisches Partizip vorliegt. Es versteht sich, dass ein kompariertes oder ein durch un- präfigiertes Partizip II (das verwöhntere Kind, das ungemachte Bett) klar adjektivisch ist. Wie verhält es sich aber mit dem pränominalen Partizip II, das in morphologischer Hinsicht nicht als Adjektiv gekennzeichnet ist? Aussagekräftig ist hier die Verwendung von Adverbialen. In Rapp (1996) wurde dargelegt, dass eine Adjektivierung des Partizip II fast ausschließlich bei telischen Verben auftritt, wobei der Resultatszustand herausgegriffen wird. Modaladverbiale wie in (19) und Agensphrasen wie in (20) sind beim adjektivierten Partizip nur möglich, wenn sie diesen Resultatszustand charakterisieren:
a. *Die Suppe ist mit dem Löffel / langsam gegessen.
b. Die Haare sind schlampig gekämmt.
a. *Die Suppe ist von einem Kind gegessen.
b. Das Bild ist von einem Kind gemalt.

Es ist davon auszugehen, dass adjektivische Partizipien den gleichen Restriktionen unterliegen, wenn sie in pränominaler Position auftreten. Folglich liefert das Vorkommen rein ereignisbezogener Modifikatoren Evidenz dafür, dass tatsächlich ein verbales Partizip II vorliegt:
a. die mit dem Löffel / langsam gegessene Suppe
b. die von einem Kind gegessene Suppe

Um sicherzustellen, dass wirklich ein verbales Partizip II vorliegt, untersuchen wir im Folgenden also stets Beispiele, die eine rein ereignisbezogene Modifikation enthalten.

Betrachten wir zunächst telische Verben (achievements und accomplishments) und deren Kombination mit Zeitadverbialen. Unsere These besagt, dass die Ereigniszeit beim verbalen Partizip II stets innerhalb der Topikzeit liegt. Dies führt dazu, dass bei Punktadverbialen nur (quasi)punktuelle Basisverben möglich sind: 
(22) achievement

a. das um 12 Uhr von Peter gekaufte Buch

b. der um 12 Uhr von Peter freigelassene Gefangene

c. das um 12 Uhr sanft eingeschlafene Kind

(23) accomplishment

a. ?das um 12 Uhr von Peter angestrichene Häuschen

b. ?das um 12 Uhr von Peter gemalte Bild

c. ?die um 12 Uhr von Peter geschriebene Dissertation

d. ?die um 12 Uhr schnell verwelkte Blume

Punktuelle Verben wie kaufen erlauben eine Inklusion der Ereigniszeit in ein quasi-punktuelles Intervall wie um $12 \mathrm{Uhr}$. Bei durativen Verben wie malen ist eine derartige Inklusion nicht möglich, was die Beispiele unter (23) abweichend macht. Zeitraumsetzende Adverbiale sind beim Partizip II dagegen mit achievements und accomplishments möglich:

(24) achievement

a. das gestern von Peter gekaufte Buch

b. der gestern von Peter freigelassene Gefangene

c. das gestern sanft eingeschlafene Kind

(25) accomplishment

a. das gestern von Peter angestrichene Häuschen

b. das gestern von Peter gemalte Bild

c. die gestern von Peter geschriebene Dissertation

d. die gestern schnell verwelkte Blume

Durch den perfektiven Aspekt des Partizip II ergibt sich, dass die Ereigniszeit auf jeden Fall innerhalb von gestern - als Maximalgröße der Topikzeit - liegen muss:

$$
\begin{aligned}
& \text { [ ------- ] } \\
& \text { gestern }
\end{aligned}
$$

Perfektivität gilt selbst für Fälle wie (25c), bei denen unser Weltwissen eine derartige Interpretation sehr unwahrscheinlich macht. Von diesen accomplishment-Fällen zu unterscheiden sind Beispiele, die zwar auch ein länger dauerndes Ereignis voraussetzen, bei denen das Verb selbst aber als achievement - nur dessen Kulminationspunkt bezeichnet: 

a. die gestern von Peter fertiggeschriebene Dissertation
b. der gestern qualvoll verdurstete Gefangene

Hier ist durch das Partizip II lediglich gefordert, dass der Kulminationspunkt innerhalb der Topikzeit liegt.

Ein Problem für die These, dass das Partizip II stets perfektiven Aspekt besitzt, stellen atelische Basisverben dar. Obwohl sie eine zeitliche Ausdehnung haben, sind sie mit Punktadverbialen möglich:
a. activity: die um 12 Uhr von zwei Kindern gezogene / getra- gene / gestreichelte Kuh
b. state: der um 12 Uhr von dem Mädchen bewunderte Filmstar

Das Partizip II scheint hier die gleichen aspektuellen Eigenschaften wie das Partizip I zu haben:
a. activity:
die um 12 Uhr eine Kuh ziehenden / tragenden / streichelnden Kinder
b. state: das um $12 \mathrm{Uhr}$ den Filmstar bewundernde Mäd- chen

Die Topikzeit wird jeweils von der Ereigniszeit inkludiert:

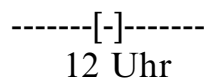

Entscheidend ist jedoch, dass das Partizip II eine derartige "Nichtperfektivität" nur bei atelischen Verben erlaubt. Was ist nun der ereignisstrukturelle Unterschied zwischen atelischen und telischen Verben? Atelische Verben sind homogen: jedes Teilintervall eines Streichelns ist wieder ein Streicheln. Telische Verben sind inhomogen: die Teilintervalle eines Verdurstens können nicht selbst als Verdursten bezeichnet werden, vgl. hierzu Dowty (1979). Die Perfektivität des Partizip II muss folglich so verstanden werden, dass ein Ereignis der genannten Art in der Topikzeit zur Gänze enthalten ist. Dies ist bei telischen Verben nur der Fall, wenn das Gesamtereignis beendet ist (starker perfektiver Aspekt), im Falle atelischer Verben jedoch auch, wenn das Gesamtereignis noch im Gange ist (schwacher perfektiver Aspekt). ${ }^{11}$

11. Marillier (1994: 23) spricht dem Partizip II das Merkmal "perfektiv" gänzlich ab und führt perfektive Interpretationen ausschließlich auf die Aktionsart des Basisverbs zurück. Eine derartige Analyse kann jedoch die offensichtlichen Unterschiede zwischen dem Partizip I und dem Partizip II bei telischen Verben nicht erfassen. 
Fazit: Das Partizip I besitzt imperfektiven Aspekt, d. h. das Ereignis wird als im Verlauf befindlich beschrieben. Das Partizip II besitzt perfektiven Aspekt; dies bedeutet, dass telische Ereignisse innerhalb der betrachteten Zeit als Ganzes enthalten sind, atelische dagegen nicht notwendigerweise.

\subsection{Temporalität}

Bisher haben wir Beispiele betrachtet, bei denen die Topikzeit des Partizips durch ein Zeitadverbiale - also auf lexikalische Weise - restringiert ist. Die Topikzeit finiter Verben ist nun zudem auf grammatische Weise bestimmt. Wir werden in diesem Abschnitt zeigen, dass bei den Partizipien eine grammatische Fixierung der Topikzeit durch Tempus nicht vorliegt; vielmehr ist ihre temporale Einordnung durch pragmatische Faktoren bestimmt.

2.2.1. Temporalität beim attributiven Partizip I. Nach Klein (1994) ist Tempus die durch grammatische Mittel festgelegte Beziehung zwischen Äußerungs- und Topikzeit. Anhand von Beispielen wie (31) zeigt Klein (1994: 222), dass das attributive Partizip I keine derartige Beziehung etabliert:
a. Paul sitzt neben einem quengelnden Kind.
b. Paul saß neben einem quengelnden Kind.

In (31a) liegt die Äußerungszeit innerhalb der Topikzeit des Partizip I, in (31b) folgt sie ihr. Eine Paraphrase der Partizipialphrasen durch Relativsätze macht dies deutlich:
a. Paul sitzt neben einem Kind, das quengelt.
b. Paul saß neben einem Kind, das quengelte.

Grund für den Unterschied ist ganz offensichtlich, dass in (31a) ein Matrixverb im Präsens, in (31b) dagegen ein Matrixverb im Präteritum vorliegt. Offenbar lehnt sich die temporale Interpretation des Partizip I an das Matrixprädikat an. Ist nun die Topikzeit des Partizips einfach mit der Topikzeit des Matrixprädikats gleichzusetzen? In diesem Falle bestünde zwar keine direkte, jedoch immerhin eine über das Matrixverb vermittelte Tempusrelation zur Äußerungszeit; die Topikzeit wäre folglich durch grammatische Mittel (finite Flexion des Matrixverbs und Partizipialmorphologie) restringiert. $\mathrm{Zu}$ erwarten wäre dann, dass das attributive Partizip I immer als gleichzeitig bzgl. des Matrixprädikats inter- 
pretiert werden muss. Gegen eine solche Betrachtung sprechen zuallererst die bereits unter 2.1. besprochenen Fälle, bei denen die Topikzeit des Partizip I durch ein Zeitadverbiale festgelegt ist. So ist es zwar durchaus möglich, dass ein Adverbiale die Gleichzeitigkeitsrelation bzgl. des Matrixprädikats verstärkt (33-1), es kann sie jedoch auch verhindern, indem es das Partizipialereignis explizit in die Vergangenheit (33-2) oder in die Zukunft (33-3) verlegt:

(33-1) Prognosen zufolge wird der Klimawandel wegen geringer Niederschläge im nördlichen und südlichen Afrika die bereits jetzt herrschende Wasserknappheit verschärfen.

http://www.venro.org/640.html (04. 07. 2011)

(33-2) Da wäre ein Ministerpräsident hilfreich, der patriotische Leidenschaft wecken könnte anstatt das Unrecht der einst herrschenden Genossen unter den Teppich zu kehren.

http://www.pnn.de/titelseite/186543/ (04. 07. 2011)

(33-3) Auf der Grundlage von Geburtsrecht und Gefährtentum verbinden sich die Heranwachsenden im Hofdienst zur später herrschenden Gruppe. http://www.thorbecke.de/ erziehung-zur-maennlichkeit-p-1340.html (04. 07. 2011)

Wie verhält es sich aber mit dem Partizip I, wenn keine Modifikation durch Adverbiale vorliegt? Man könnte zunächst annehmen, dass die temporale Interpretation zumindest hier immer in Anlehnung an das Matrixprädikat erfolgt, und zwar im Sinne einer Gleichzeitigkeitsrelation. Nun darf bei der Betrachtung attributiver Partizipien nicht vernachlässigt werden, dass sie sich nicht direkt mit dem Matrixprädikat verbinden, sondern Modifikatoren eines Nomens sind. Dies verlangt einige generelle Überlegungen zur temporalen Interpretation von Nominalphrasen. ${ }^{12}$ Enç (1986) stellt fest, dass Nomina eine vom Matrixprädikat unabhängige temporale Interpretation haben können. Musan (1997) präzisiert diese Einsicht, indem sie die Abhängigkeit vom jeweils auftretenden Determinans analysiert. So besitzen nur Nominalphrasen, deren Determinans eine Existenzpräsupposition auslöst (wie der definite Artikel, das Possessivum, das Demonstrativum), eine temporal unabhängige Interpretation:

Die Lehrerin ging in Brooklyn zur Schule.

12. Wir danken Klaus von Heusinger für Anregungen bzgl. der temporalen Interpretation von Nominalphrasen. 
Beispiel (34) bedeutet, dass eine Person in Brooklyn zur Schule ging, die zu einem späteren Zeitpunkt ihres Lebens Lehrerin wurde. In Nominalphrasen mit indefinitem Artikel - in denen keine Existenzpräsupposition vorliegt - ist eine derartige unabhängige temporale Interpretation dagegen nicht möglich: ${ }^{13}$

?Eine Lehrerin ging in Brooklyn zur Schule.

Pränominale Partizipialphrasen werden nun wie Adjektive mit dem Nomen durch Intersektion verknüpft, dabei entsteht eine komplexe Eigenschaft:

a. (der) müde Mann = jemand, der ein Mann ist und müde ist

b. (der) brüllende Mann = jemand, der ein Mann ist und brüllt

Nominaleigenschaft und Adjektiv- bzw. Partizipialeigenschaft müssen immer überlappen, d.h. (36) kann keinesfalls so interpretiert werden, dass jemand, der zu einer bestimmten Zeit ein Mann ist, zu einer anderen Zeit - also z. B. als Baby - brüllt bzw. müde ist. ${ }^{14} \mathrm{Ob}$ eine vollständige temporale Übereinstimmung zwischen Nominaleigenschaft und Adjektiv- bzw. Partizipialeigenschaft vorliegt, ist dagegen nicht grammatisch vorgegeben. Es gibt jedoch bestimmte Tendenzen der Inklusion, die auf die prototypischen temporalen Eigenschaften der lexikalischen Kategorien zurückgeführt werden können. Wie Lehmann (1992: 158) vor-

13. Die folgenden Beispiele scheinen auf den ersten Blick gegen die These zu sprechen, dass indefinite Nominalphrasen immer temporal abhängig von der Matrixtopikzeit sind:

(i) Ich kenne eine Lehrerin, die in Brooklyn zur Schule ging.

(ii) Ich kenne eine Lehrerin, die ging in Brooklyn zur Schule.

Die temporale Verschiebung in (i) betrifft nun allerdings nicht die Matrixtopikzeit, sondern den Relativsatz. Wie in Kusumoto (2005) dargelegt, sind Relativsätze generell temporal unabhängig - dies betrifft sowohl ihr Verhältnis zum Matrixsatz als auch zum modifizierten Nomen. Dabei spielt es keine Rolle, ob sie in definiten oder indefiniten Nominalphrasen auftreten. In (ii) liegt eine Parataxe vor; hier ist nicht die indefinite Nominalphrase eine Lehrerin, sondern das als definites Pronomen verwendete die temporal unabhängig von seiner Matrixtopikzeit. Weder (i) noch (ii) spricht also gegen die temporale Abhängigkeit indefinite Nominalphrasen von ihrer Matrixtopikzeit.

14. Angemerkt sei, dass Adverbiale in bestimmten Fällen eine Dissoziation von Partizipialereignis und Nominaleigenschaft bewirken können:

(i) Der früher selbst rauchende Präsident setzte sich nach seinem Amtsantritt für Nichtrauchergesetze ein.

(i) kann auf zweierlei Weise interpretiert werden: zum einen ist es möglich, dass der Präsident vor der Äußerungszeit rauchte, zum anderen jedoch auch, dass ein bestimmtes Individuum rauchte, bevor es Präsident wurde. In der zweiten Lesart werden Partizipialereignis und Nominaleigenschaft durch früher getrennt. Auf derartige Fälle können wir hier jedoch nicht weiterführend eingehen. 
schlägt, können die Kategorien folgendermaßen auf einem Kontinuum angeordnet werden: ${ }^{15}$

Tabelle 2: Prototypische temporale Eigenschaften lexikalischer Kategorien

\begin{tabular}{|l|ccc|}
\hline Begriff & Gegenstand & Eigenschaft & Ereignis \\
\hline $\begin{array}{l}\text { Zeitstabilität } \\
\text { Funktion }\end{array}$ & maximal & $\leftarrow----------------\rightarrow$ & $\begin{array}{c}\text { minimal } \\
\text { Prädikation }\end{array}$ \\
\hline Wortart & Substantiv & Attribution & Verb \\
\hline
\end{tabular}

Wird nun eine komplexe Eigenschaft aus Nomen und Adjektiv gebildet, so ist im prototypischen Fall die nominale Eigenschaft von längerer Dauer als die adjektivische (36a). In verstärktem Maße gilt dieses Ungleichgewicht für die Verbindung von Nomen und Partizip I, weil das Partizip der Kategorie Verb angehört: In (36b) bezeichnet das Nomen Mann die in hohem Maße zeitstabile Eigenschaft eines Individuums, brüllend dagegen benennt ein im Normalfall nur kurze Zeit andauerndes Ereignis. Ein Partizip I gilt demnach im unmarkierten Falle für ein wesentlich kürzeres Zeitintervall als das Nomen, mit dem es sich verbindet. Es gibt jedoch durchaus auch Fälle, bei denen die beiden Eigenschaften als deckungsgleich interpretiert werden. Erwartungsgemäß ist dies der Fall bei ereignisbezeichnenden Nomina, die - wie Verben - ein Minimum an Zeitstabilität aufweisen: ${ }^{16}$
a. der die Zuschauer gefährdende Sturz / Sprung
b. der die Menschen erschreckende Salto / Blitz / Donner

Unsere Frage ist nun, wie sich eine aus Partizip I und Nomen bestehende komplexe Eigenschaft (brüllender Mann) bzgl. des Matrixprädikats verhält. ${ }^{17}$ Tritt sie innerhalb einer definiten Nominalphrase auf, so besteht temporale Unabhängigkeit gegenüber dem Matrixprädikat. Betrachten wir folgendes Beispiel:

15. Vgl. zum Konzept der Zeitstabilität auch Givón (1984) und Lenz (1993).

16. Ereignisbezeichnende Nomina besitzen sehr häufig, jedoch nicht immer eine verbale Basis: (37a) vs. (37b).

17. Die folgenden Überlegungen gelten auch für komplexe Eigenschaften, die sich aus einem Adjektiv und einem Nomen zusammensetzen: So kann in den Beispielen (38a)(38c) anstelle des Partizip I auch ein Adjektiv wie laut verwendet werden, das zur Matrixtopikzeit nicht mehr gilt, jedoch die Identifizierung des Mannes sichert. Tendenziell gilt jedoch, dass das Partizip I als Verbform für derartige Lesarten aufgrund seiner temporalen Instabilität besser geeignet ist als ein genuines Adjektiv. 
Ich beobachtete einen Mann, der auf dem Hof herumschrie und dann davonlief.

a. Wenig später sah ich den brüllenden Mann friedlich in einer Zeitung lesen.

b. Kurz zuvor hatte ich den brüllenden Mann noch friedlich im Hof stehen sehen.

c. Als Kind hatte der brüllende Mann mit meiner Tochter Sandkuchen gebacken.

In den Fortführungssätzen (38a)-(38c) wird durch der brüllende Mann jeweils ein Individuum bezeichnet, das zu einer bestimmten - in diesem Fall durch den Einleitungssatz vorgegebenen - Zeit brüllt und ein Mann ist. In (38a) und (38b) gilt die Eigenschaft Mann-Sein zur Matrixtopikzeit, nicht jedoch die Eigenschaft brüllen - in beiden Fällen besteht eine Unverträglichkeit zum Adverbiale friedlich, das Attribut brüllend sichert lediglich die Identifizierung des Mannes. In (38c) gilt keine der beiden Eigenschaften zur Matrixtopikzeit. Da der Bedeutungsbestandteil, den die Partizipialphrase beisteuert, im Allgemeinen weniger zeitstabil ist als derjenige, den das Nomen liefert, sind temporale Verschiebungen wie in (38a) und (38b) wesentlich häufiger anzutreffen als solche wie in (38c). Man kann jedoch auch Belege finden, bei denen das Partizip eine Eigenschaft angibt, die zur Matrixtopikzeit noch gilt, das Nomen dagegen nicht:

(39-1) Philipp Benz ist mit 98 Jahren nicht nur der letzte noch lebende Häftling des frühen Konzentrationslagers Osthofen, er ist als Zeitzeuge auch ein profunder Kenner des antifaschistischen Widerstands und der politischen Nachkriegsentwicklung in seiner Region.

http://www.frankfurter-info.org/Nachrichten/ zeitzeugnisse-als-broschure-erschienen-philipp-benzals-historiker (04. 07. 2011)

Bezeichnenderweise basiert das Partizip lebend auf einem hochgradig zeitstabilen Zustandsverb, Häftling dagegen bezeichnet die im Normalfall vorübergehende Eigenschaft eines Individuums.

Die Beispiele (38) und (39) zeigen, dass kontextuelle Faktoren für die Geltungszeit des Partizips (und des Nomens) bei definitem Determinans eine Rolle spielen. Interessant ist, dass der imperfektive Aspekt des Partizip I auch bei einer Vorzeitigkeitsinterpretation erhalten bleiben muss. Um dies zu illustrieren, wählen wir ein Kreationsverb (schreiben) und ein Konsumverb (trinken). Stellen wir uns folgende Situationen vor:

(40) Schulsituation. Der Lehrer findet auf seinem Platz einen Umschlag, der einen an ihn gerichteten Brief mit Schimpfwörtern enthält. Er liest ihn, hält ihn hoch und sagt: 
?Der diesen Brief schreibende Schüler muss mit einem harten Verweis rechnen.

(41) In einer Kneipe. Kellner hält ein leeres Bierglas hoch und fragt: ?Hat der dieses Bier trinkende Gast bereits gezahlt?

Der Kontext stellt in (40) klar, dass der Brief schon fertiggestellt ist. Aus diesem Grund ist die Verwendung des Partizip I - welches Imperfektivität und damit Nichtvollendung des Objekts ausdrückt - abweichend. Ebenso kann das Partizip I nicht in Verbindung mit einem Objekt verwendet werden, das - wie das Bier in (41) - bereits völlig konsumiert ist. Dies zeigt, dass die aspektuelle Markierung als imperfektiv dem Partizip I inhäriert und durch eine temporale Verschiebung nicht aufgehoben werden kann.

Neben Fällen, bei denen die temporale Interpretation definiter Nominalphrasen kontextuell bestimmt ist, gibt es auch solche, bei denen auf die Äußerungszeit zugegriffen wird (= temporal-deiktische Interpretation):

(42-1) Die Mehrzahl der Asylbegehren der in Solingen lebenden Flüchtlinge ist also bereits abgelehnt worden. Diese müssen daher mit einer Abschiebung rechnen.

http://home.wtal.de/tacheles-Solingen/aktuell/ausg38/ seite08.html (04. 07. 2011)

Wir verstehen (42-1) im Normalfall so, dass die Flüchtlinge zur Äußerungszeit in Solingen leben, während nichts darüber ausgesagt wird, wo sie sich aufgehalten haben, als ihre Asylbegehren abgelehnt wurden.

Eine formal-semantische Analyse definiter Determinantien und ihres Einflusses auf die temporale Interpretation von Nomina und deren Attributen kann im Rahmen dieses Aufsatzes nicht erfolgen. ${ }^{18}$ Intuitiv ist es jedoch durchaus einsichtig, dass temporal unabhängige Lesarten definite Determinantien erfordern: anaphorischer Bezug auf etwas Vorerwähntes ist nur durch eine definite Nominalphrase möglich, ebenso erfordert temporal-deiktischer Zugriff ein Demonstrativum bzw. einen definiten Artikel. Halten wir also fest, dass Einbettung in eine definite Nominalphrase eine vom Matrixprädikat unabhängige temporale Interpretation des Partizip I möglich macht.

$\mathrm{Zu}$ überlegen ist nun allerdings, warum auch bei definitem Determinans eine attributive Partizipialphrase im unmarkierten Fall als gleich-

18. Vgl. hierzu Enç (1986), Musan (1997) und Kusumoto (2005). 
zeitig zum Matrixprädikat interpretiert wird. Die Erklärung hierfür ergibt sich auf pragmatischer Ebene aus der Maxime der Relevanz (Grice 1975). Wir gehen davon aus, dass ein Attribut für den Gesamtsatz dann relevant ist, wenn es sein Bezugsnomen zur Matrixtopikzeit charakterisiert. Das attributive Partizip I hat nun Subjektsbezug; d. h. sein Bezugsnomen entspricht dem Subjekt des zugrundeliegenden Verbs. In Arbeiten zur lexikalischen Semantik wurde schon häufig festgestellt, dass Subjektargumente im Allgemeinen durch das Verbalereignis keine Zustandsveränderung erfahren (vgl. Rapp 1997): So wird bei der großen Gruppe der telischen transitiven Verben das Objekt, nicht jedoch das Subjekt in einen Resultatszustand versetzt (zerstören, bauen, essen, befreien), bei der kleineren Gruppe der atelischen transitiven Verben (streicheln) keines der beiden Argumente. Auch die intransitiven haben-Verben (arbeiten, tanzen) versetzen ihr Subjekt nicht in einen Resultatszustand. Der Nachzustand des Ereignisses ist also für die Charakterisierung des Subjektarguments in all diesen Fällen nicht geeignet. Hieraus ergibt sich, dass die einzige Ereignisphase, durch die das Subjekt charakterisiert werden kann, die Verlaufsphase des Verbs ist - genau dies führt zur Gleichzeitigkeitsinterpretation bzgl. des Matrixprädikats.

Aufschlussreich sind in diesem Zusammenhang Verben wie gewinnen und verlieren, die eine Zustandsveränderung benennen, von der neben dem Objekt auch das Subjekt betroffen ist. Hier sind Beispiele mit einer Vorzeitigkeitsinterpretation des Partizip I leicht zu finden:

(43-1) Die beiden verlierenden Mannschaften Schweden und Dänemark bestritten am 17. Mai 2009 ein Spiel um den 17. und 18. Platz. http://de.wikipedia.org/wiki/Sportkegel-Weltmeisterschaft_2009

(04. 07. 2011)

(43-2) ... aber wahrscheinlich werden diese Obermilchmädchen von der FIFA diesen durchgeknallten Schiri noch belohnen, wenn Spanien ausscheidet. Dann darf der noch ein Viertelfinale pfeifen und dafür sorgen, dass das gewinnende Team zum Halbfinale dann nur noch mit 8 Feldspielern auflaufen darf. http://www.tagesspiegel.de/sport/fussball-wm2010 (04. 07. 2011)

(43-3) Wie bei den bisherigen Turnieren wurden in allen Wertungen Pokale vergeben und den gewinnenden Mannschaften zur Pflege mit nach Hause gegeben.

http://doko-fisch.de/f14/ergebnisSCH.html (04. 07. 2011)

Ausschlaggebend dafür ist, dass hier das Subjektargument durch den Nachzustand des Verbalereignisses affiziert ist - die gewinnenden bzw. 
verlierenden Mannschaften haben durch das Spiel einen bestimmten Status erreicht. ${ }^{19}$ Halten wir fest, dass ein pränominales Partizip I innerhalb definiter Nominalphrasen temporal unabhängig gegenüber dem Matrixprädikat ist, wie das Nomen selbst auch. Die unmarkierte Interpretation ist im Normalfall die Gleichzeitigkeitsinterpretation, die als eine generalisierte konversationelle Implikatur auf der Basis der Relevanzmaxime entsteht. Spezifische Kontexte wie in (38), (39) und (42) sowie spezifische Basisverbklassen wie in (43) verhindern das Aufkommen dieser Implikatur.

Fazit: Der Aufbau von Temporalität beim pränominalen Partizip I erfolgt kompositional "von innen nach außen". ${ }^{20}$ Ist das Partizip durch Zeitadverbiale modifiziert, so werden diese bei der Bedeutungsberechnung unmittelbar miteinbezogen. Liegt dagegen keine lexikalische Restriktion der Topikzeit durch Adverbiale vor, so wird im unmarkierten Fall unter Rekurs auf die Maxime der Relevanz Gleichzeitigkeit mit der Matrixtopikzeit angenommen. Diese Gleichzeitigkeitsimplikatur kann im Falle definiter Nominalphrasen jedoch verhindert werden, wenn ein deiktischer Gebrauch mit direktem Bezug auf die Sprechzeit vorliegt oder das Partizip I in identifizierender Funktion mit Bezug auf einen vorerwähnten Kontext verwendet wird.

2.2.2. Temporalität beim attributiven Partizip II. Auch das attributive Partizip II wird im unmarkierten Fall durch Bezug auf das Matrixprädikat temporal verankert, allerdings geht die Topikzeit des Partizip II derjenigen des Matrixprädikats zumeist voraus. Ausschlaggebend für diesen Unterschied zum Partizip I ist die Affiziertheit des Bezugsnomens durch das Partizipialereignis. Bei der Mehrzahl der transitiven Verben (z. B. zerstören, bauen) erfährt das Objekt eine Zustandsveränderung durch das Verbalereignis - dieses Objekt ist zugleich Bezugsnomen des Partizip II. Gleiches gilt für die intransitiven sein-Verben, deren Partizip II pränominal verwendet wird (einschlafen, einstürzen): Auch hier ist das Bezugsno-

19. Auch bei den intransitiven sein-Verben erleidet das Subjekt eine Zustandsveränderung. Dennoch wird das Partizip I hier nie als vorzeitig interpretiert. Der Grund dafür liegt in der Konkurrenz durch das Partizip II, das bei dieser Verbgruppe ebenfalls Subjektbezug hat und kanonisch als vorzeitig interpretiert wird.

20. In einem Gutachten wurde darauf hingewiesen, dass der Aufbau der Temporalität beim pränominalen Partizip kompositional erfolgt und dass keiner der Interpretationsschritte (von innen nach außen) revidiert wird. Dies schließt jedoch u.E. nicht aus, dass ab einem bestimmten Schritt pragmatische Prinzipien - in diesem Falle generalisierte konversationelle Implikaturen - eine Rolle für die Festlegung der Topikzeit spielen. Eine lexikalische Spezifizierung der Topikzeit durch Zeitadverbiale wird hierdurch jedoch tatsächlich niemals aufgehoben. 
men des Partizip II durch das Verbalereignis affiziert. Wir gehen nun davon aus, dass die einem Nomen durch Attribution zugeschriebene Eigenschaft als möglichst zeitstabil interpretiert wird..$^{21}$ Das Partizip II hat zwar verbalen Charakter, ist also per se minimal zeitstabil - sein Auftreten in einer Adjektivposition führt jedoch dazu, dass im Falle einer Wahlmöglichkeit diejenige Lesart herausgegriffen wird, die die größere Zeitstabilität garantiert. Charakterisierung durch einen Resultatszustand ist nun eindeutig zeitstabiler als Charakterisierung durch die Verlaufsphase eines Verbs. Konsequenterweise interpretieren wir ein pränominales Partizip II - sofern das Basisverb telisch ist - als vorzeitig bzgl. der Topikzeit des Matrixprädikats, vgl. (44). Nur bei der kleinen Gruppe der atelischen transitiven Verben - die ja das Objekt nicht in einen Resultatszustand versetzen - wird Charakterisierung durch die Verlaufsphase (und damit eine Gleichzeitigkeitsinterpretation) vorgezogen, vgl. (45): 22
a. Er blickte auf die zerstörte Stadt.
b. Die zerstörte Stadt lag in einem Tal.
c. Peter wohnte neben der eingestürzten Eishalle. ${ }^{23}$

a. Er betrachtete den von zwei Männern gezogenen Wagen.
b. Sie erblickte die von einem Kind gestreichelte Katze.

Allerdings gibt es auch beim Partizip II telischer Verben spezifische Kontexte, bei denen keine Vorzeitigkeit bzgl. der Matrixtopikzeit, sondern einer anderen kontextuell gegebenen Zeit - zumeist der Äußerungs-

21. Ein Gutachter hat uns darauf hingewiesen, dass das Erfordernis der Zeitstabilität für Relativsatzattribute nicht gilt. U. E. ist dies darauf zurückzuführen, dass durch die finite Tempusflexion hier eine direkte Anbindung an die Äußerungszeit besteht (vgl. Kusumoto 2005): Der Relativsatz schreibt also einem Individuum eine Eigenschaft zu, die zu einer bestimmten, durch das Tempus spezifizierten Zeit gilt. Eine derartige Festlegung besitzen Adjektiv- und Partizipialattribute - mangels Tempusflexion - nicht. Die temporale Interpretation wird bei ihnen dadurch geleitet, dass das attribuierte Nomen in essentieller, d. h. möglichst zeitstabiler Weise charakterisiert wird. Aufschlussreich ist hierbei auch, dass die Partizipialeigenschaft, nicht jedoch die Relativsatzeigenschaft, in jedem Falle als kotemporär zur Nominaleigenschaft interpretiert werden muss:

(i) *die hier zur Grundschule gehende Politikerin

(ii) die Politikerin, die hier zur Grundschule ging

22. Nach Struckmeier (2007: 36) verlangt die Attribution allerdings einen weniger hohen Grad an Zeitstabilität als die primäre Prädikation.

23. (44b) und (44c) zeigen deutlich, dass für die temporale Verankerung des Partizipialereignisses nur die Topikzeit des Matrixprädikats, nicht aber dessen Ereigniszeit relevant ist. So ist es in (44c) durchaus möglich, dass Peter nicht nur nach dem Einsturz, sondern auch zuvor und währenddessen neben der Eishalle wohnte, betrachtet wird jedoch nur eine Zeit nach dem Einsturz. 
zeit - besteht. Voraussetzung für derartige temporal unabhängige Interpretationen ist wiederum die Einbettung des Partizips in eine definite Nominalphrase:

(46-1) Die nun in Smolensk abgestürzte Maschine habe die auf den TU154-Bau spezialisierte Werkstatt in tadellosem Zustand verlassen.

http://www.zeit.de/gesellschaft/zeitgeschehen/2010-04/ flugzeugabsturz-praesident-polen (04. 07. 2011)

(46-2) Der ermordete Politiker war ein enger Verbündeter des Präsidentschaftskandidaten Luis Inacio.

http://www.shortnews.de/id/346901/

Brasilien-Buergermeister-von-Kugeln-durchsiebt (04. 07. 2011)

Auch (44c) lässt einen deiktischen Bezug des Partizips auf die Äußerungszeit zu: Peter kann neben einer Eishalle gewohnt haben, die damals noch intakt war, jedoch inzwischen - d.h. vor der Äußerungszeit eingestürzt ist.

Ebenso wie das Partizip I kann das Partizip II zudem durch Zeitadverbiale explizit temporal bestimmt werden. Die Partizipialphrase in (47-1) wird durch später als nachzeitig zur Matrixtopikzeit eingeordnet:

(47-1) So hat er, um beim 15. Juli zu bleiben, auch die Herkunftsorte der später vor Gericht gestellten Verhafteten detailliert aufgeschlüsselt, ihre soziale Herkunft, ihre Berufe, natürlich Alter und Geschlecht.

In seltenen Fällen können Adverbiale sogar eine Gleichzeitigkeitsinterpretation bzgl. der Matrixtopikzeit erzeugen: ${ }^{24}$
a. Ohne sich zu bewegen, starrte Paul auf das vor seinen Augen erwürgte Kaninchen.
b. Gebannt starrte ich auf den vor meinen Augen verzehrten Ku- chen.

Bemerkenswert ist, dass der perfektive Aspekt des Partizip II trotz Gleichzeitigkeitsinterpretation erhalten bleibt. Das Verbalereignis wird als insgesamt abgeschlossen betrachtet; im Gegensatz zum Partizip I ist

\footnotetext{
24. Bei intransitiven sein-Verben (einschlafen, einstürzen) lässt sich keine Gleichzeitigkeitsinterpretation des pränominalen Partizip II erzwingen. Der Grund dafür scheint zu sein, dass in diesem Falle das Partizip I, das ja den gleichen Argumentbezug aufweist, vorgezogen wird.
} 
keine Situation vorstellbar, in der der Kulminationspunkt nicht erreicht wird:

(49) a. Man entdeckte den verdurstenden Mann noch rechtzeitig.

b. *Man entdeckte den verdursteten Mann noch rechtzeitig.

(50) a. Der Giftpfeil traf die ein Kaninchen erwürgende Schlange noch rechtzeitig.

b. *Der Giftpfeil rettete das von einer Schlange erwürgte Kaninchen noch rechtzeitig.

Bei Verben, deren Resultatszustand aufgehoben werden kann, liegt in entsprechenden Kontexten eine restitutive Lesart vor:

\section{Max fand seinen verlorenen Schlüssel noch rechtzeitig.}

Wir können (51) nur so interpretieren, dass der bereits vollständig verlorene Schlüssel wiedergefunden wurde, nicht aber so, dass das Ereignis des Verlierens hier unterbrochen wird. All dies zeigt sehr deutlich, dass der perfektive Aspekt - im Gegensatz zur temporalen Interpretation eine inhärente und nicht tilgbare Eigenschaft des Partizip II ist.

Fazit: Auch beim Partizip II ist Tempus nicht grammatisch kodiert; eine Topikzeit kann auf ganz verschiedene Weise geliefert werden. Zeitadverbiale können die Topikzeit auf lexikalischem Wege restringieren. Tritt kein Zeitadverbiale auf, so ergibt sich bei telischen Verben eine Vorzeitigkeit gegenüber der Matrixtopikzeit als konversationelle Implikatur; eine solche kann jedoch in spezifischen Kontexten - insbesondere bei deiktischem Gebrauch mit direktem Bezug zur Sprechzeit - verhindert werden.

\section{Argumentstruktur}

\subsection{Argumentadaptionen}

Im vorigen Abschnitt haben wir gezeigt, dass pränominale Partizipien hinsichtlich des Aspekts grammatisch festgelegt sind, während ihre temporale Interpretation durch verschiedenartige kontextuelle Faktoren bestimmt wird. Im Folgenden geht es um die Interpretation von attributiven Partizipien hinsichtlich ihrer Argumentstruktur.

Laut gängigen Grammatiken ist die attributive Verwendung eines Partizips abhängig von den syntaktischen Eigenschaften seines Basisverbs. Für ein Partizip I muss die Argumentstruktur des zugrunde liegenden 
Verbs ein Subjekt bereitstellen, das dann in der Position des Bezugsnomens erscheint, vgl. (52a). Im Falle des Partizip II entspricht das Bezugsnomen bei transitiven Verben dessen direktem Objekt, vgl. (52b).
a. der lesende Schüler
b. das gelesene Buch

Wie bereits in Abschnitt 1 erwähnt, liegt mit der Attribution des Partizips eine Merkmalsbündelung vor: im Partizip I ist der Subjektbezug mit imperfektiver Aspektualität kombiniert, im Partizip II der Objektbezug mit perfektiver Aspektualität. Eine Auflösung dieser Merkmalsbündelung ist nur dann gegeben, wenn das Partizip auf ein intransitives seinVerb zurückgeht. In diesem Falle kommt allein die Aspektopposition zwischen den Partizipien zum Tragen. Das Bezugsnomen ist sowohl für das Partizip I als auch für das Partizip II das Subjekt des Basisverbs:

\section{der verblühende / verblühte Flieder}

Um die (wie im Standardfall unter (52a) und (52b)) vorhandene Merkmalsbündelung von Aspektualität und Argumentbezug zu lösen, existiert für die pränominale Attribution eine alternative Ausdrucksmöglichkeit. Sie besteht aus dem Partizip I des Passiv- bzw. Perfektauxiliars (werden bzw. haben), das jeweils um das betreffende Partizip II erweitert ist:

$$
\begin{aligned}
& \text { a. der gelesen habende Schüler } \\
& \text { b. das gelesen werdende Buch }
\end{aligned}
$$

(Subjektbezug / perfektiv)

(Objektbezug / imperfektiv)

Damit sind alle möglichen Kombinationen dieser beiden Merkmale abgedeckt, die Bildungen in (54) gelten jedoch als schwerfällig und selten, einige Grammatiken stufen sie sogar als ungrammatisch ein.

Wie die Ergebnisse unserer Korpusrecherche zeigen, gibt es daneben eine Reihe von Bildungen, in denen auch das Partizip II eines intransitiven Verbs in pränominaler Attribution vorkommt:

(55-1) Das Verhalten der exilierten deutschen Wissenschaftler, die angebotenen Stellen mit dem Wissen der vorher stattgefundenen Entlassung trotzdem anzunehmen, stößt teilweise noch heute auf Kritik.

(55-2) Bei dieser Weltbesichtigung hatte der Reporter Scholl-Latour Gefahren zu bestehen, wie wir sie nur aus den geschwindelten Abenteuern Karl Mays kennen. 
(55-3) Nach im Gewerbe umlaufenden, nicht widersprochenen Berichten hat er den Anstalten "Barbarei im kollegialen Zusammenleben" vorgeworfen.

Da die oben beschriebene Merkmalsbündelung von Aspektualität und Argumentbezug semantisch im Partizip verankert ist, stellen die Bildungen in (55) Regelverletzungen dar. Sie sind jedoch interpretierbar, weil es auf der pragmatischen Ebene einen Spielraum gibt, der es ermöglicht, die Argumentstruktur des zugrunde liegenden Verbs unter bestimmten Bedingungen zu ignorieren. Das, was bei der Interpretation hier zusätzlich durch eine Adaption geleistet werden muss, ist die richtige Zuordnung des Bezugsnomens zu einem Argument des zugrunde liegenden Verbs, genauer gesagt zu dessen thematischer Rolle.

Ein allgemein üblicher und naheliegender Umgang mit Daten dieser Art ist ihre Einordnung als ein Randphänomen, das für die Sprachkompetenz keine Rolle spielt und allenfalls in den Bereich der Sprachperformanz fällt, wobei diese Bildungen dann lediglich als okkasionell, schlechtes oder sogar falsches Deutsch eingestuft werden. Eine Auffassung, die implizit in normativen Grammatiken und explizit u. a. in Zimmer (2007) oder im Duden (2007) vertreten wird. Gegen eine solche Einordnung sprechen u.E. mehrere Gründe: Zum einen sind diese Vorkommen in hoher Anzahl belegbar und dabei keineswegs auf Mündlichkeit oder Umgangssprache beschränkt. So ist beispielsweise in dem von uns gewählten Korpus DIE ZEIT die Anzahl der Belege für das regelwidrige Vorkommen des attributiven Partizips II von stattfinden deutlich höher als die entsprechenden regelkonformen Alternativen. ${ }^{25}$ Zum anderen wäre zu erwarten, dass relativ willkürlich gegen die hier geltende grammatische Regel verstoßen wird. Aber das Gegenteil scheint der Fall zu sein: Es gibt nicht nur deutliche Akzeptabilitätsunterschiede zwischen den einzelnen Bildungen, sondern auch mögliche Bildungen, für die eine Adaption ausgeschlossen ist und für die sich dementsprechend auch keine Belege in dem von uns gewählten Korpus finden lassen. Darüber hinaus tritt diese Art der Regelverletzung nur in der pränominalen Attribution auf, in der Prädikation ist sie ausgeschlossen, und die beiden Partizipien sind nicht gleichermaßen davon betroffen, sie ist nur beim Partizip II zu finden. Auch diese asymmetrische Verteilung spricht dafür, dass diese Regelverletzung nicht einfach zufällig geschieht, sondern dass

25. Den 123 Belegen für das regelwidrige Bildungsmuster stattgefundene $X$ stehen nur drei Belege des regelkonformen Bildungsmusters stattgefunden habende $X$ gegenüber. Dieser Befund deckt sich mit den Ergebnissen der Recherchen von Struckmeier (2007: 11) und Pakkanen-Kilpiä (2004). Handwerker (2007: 135) vermutet, dass derartige Regelabweichungen auf dem Wege zur standardsprachlichen Akzeptanz sind. 
es bestimmte Gründe und Voraussetzungen dafür gibt, die das Sprachsystem (und damit auch die Sprachkompetenz) betreffen und aus dem heraus sie erklärt werden können.

Betrachten wir diese Grauzone zwischen Grammatik (den regulär gebildeten Formen) und Lexikon (den als Adjektiv lexikalisierten Partizipien) einmal genauer. In welchem Verhältnis können ein attributives Partizip und sein Bezugsnomen im Hinblick auf das zugrunde liegende Verb und dessen Argumentstruktur tatsächlich zueinander stehen? Welches sind die Bedingungen, die hier einen interpretatorischen Spielraum für Adaptionen eröffnen und wo liegen die Grenzen dieser Flexibilität? Dazu soll im Folgenden zunächst das Spektrum der Variation anhand der Beispiele unter (55) nach Gruppen sortiert vorgestellt und genauer erläutert werden. Von der Betrachtung ausgeschlossen werden dabei Fälle, bei denen es sich um einen metaphorischen oder metonymischen Gebrauch handelt (fragende, verzweifelte Blicke), Fälle, in denen ein als Adjektiv lexikalisiertes Partizip vorliegt, dessen Bedeutung nicht (mehr) transparent ist (bedeutender, erfahrener Lehrer) und okkasionelle Bildungen (abenteuernder Mensch), zu denen kein (etabliertes) Basisverb existiert, die aber dennoch vollständig transparent sind.

\subsection{Das Spektrum der Argumentadaptionen}

Die erste Gruppe (vgl. Beispiel (55-1) sind Bildungen, denen mit dem attributiven Partizip II ein intransitives, genauer, ein einstelliges Verb zugrunde liegt, das als Auxiliar haben selegiert. Das Partizip kann zusätzlich durch adverbiale Modifikatoren ergänzt sein:

(56-1) Die Unterdrückung der Frau, die niemals stattgefundene Reformation, die Rückständigkeit der islamisch geprägten Gesellschaften in Wissenschaft und Bürgerfreiheit, das alles muss man wirklich nicht zum hundertsten Mal schreiben.

(56-2) Eine Chance sah ich darin, dass die Frau des Hauses sich früh in der Wohnung und im Bad für ihren Dienst zurechtzumachen hatte und somit etwas Unruhe in die stagnierte Lage brächte.

(56-3) Und wenn alles nur ein großer, böser Trick war, um das bedenklich nachgelassene Interesse an Michael Jacksons CDs etwas anzuschieben?

(56-4) Dass dies keine ungebotenen Forderungen sind, beweist der seit dem Krieg ständig zugenommene Lesehunger. 
Es handelt sich hier um ein sehr gut belegbares und hinsichtlich seiner Abweichung um ein relativ unauffälliges Bildungsmuster, bei dem mit dem Partizip II in der Attribution die Merkmalskombination perfektive Aspektualität und Subjektbezug vorliegt. Die Verletzung der Argumentstruktur des zugrunde liegenden Verbs dient in diesen Fällen also dazu, aspektuell eindeutig differenzieren zu können:

(56-4') [...] der seit dem Krieg ständig zunehmende / zugenommene Lesehunger $[\ldots]$

Die notwendige Adaption wird dadurch ermöglicht, dass die jeweiligen Basisverben neben dem Subjekt über keine weiteren Argumente verfügen und die mit den Partizipien im Standardfall vorhandene Merkmalsopposition zwischen Subjekt- und Objektbezug von vornherein nicht gegeben ist. Die Interpretation des Bezugsnomens als Subjekt des zugrunde liegenden Verbs ist somit die einzig mögliche. In all diesen Bildungen wird die notwendige Adaption begünstigt durch ihre semantische Nähe zu den intransitiven sein-Verben wie einschlafen, einstürzen und verblühen (vgl. Fußnote 2). Wie bei diesen liegen hier keine prototypischen Verben vor: Sie denotieren keine Handlungen und verfügen nur über ein Argument, das nicht agentiv ist, sondern (eher wie ein prototypisches Objekt) selbst einer Veränderung unterliegt, aber syntaktisch dennoch als Subjekt realisiert wird. Diese Bildungen können somit als Analogiebildungen eingeordnet werden, denen die intransitiven sein-Verben als Muster dienen.

Wenn das betreffende Basisverb jedoch ein transitives homonymes Gegenstück besitzt, wie das für den Hörbeleg in (57) der Fall ist, ist diese Eindeutigkeit der Interpretation nicht mehr ohne Weiteres gegeben, die Regelverletzung deutlich markierter und eine Adaption folglich aufwändiger:

die eingesetzten Schneefälle

Hier dominiert zunächst die passivische Interpretation, bei der das Bezugsnomen regulär als direktes Objekt eines zugrunde liegenden transitiven Verbs gedeutet wird. Erst mit einem geeigneten Kontext (z. B. im Rahmen eines Wetterberichts) wird eine entsprechende Adaption ausgelöst und das zugrunde liegende Verb auf seine intransitive lexikalische Variante zurückgeführt. Die Adaption kann auch durch jeweils unterschiedliche adverbiale Modifikatoren unterstützt werden, vgl. die Gegenüberstellung von regulärer (58-1) vs. adaptiver (58-2) Verwendung, abgeleitet einmal aus der transitiven und einmal aus der intransitiven lexikalischen Variante des Verbs bestehen, die beide haben als Perfektauxiliar selegieren: 
(58-1) Freundschaftlich erinnert sich der Mann an die gemeinsam bestandene Gefahr: "Wir beide können doch dem Herrgott danken, daß wir noch einmal davongekommen sind."

(58-2) Die Gründe dafür dürften in erster Linie in der zeitweilig bestandenen Streikgefahr im malaiischen Zinnbergbau gelegen haben.

Darüber hinaus zeigen diese Bildungen Ähnlichkeiten zu Partizipien, die von den Grammatiken als lexikalisierte Relikte des Sprachwandels eingestuft und in Wörterbüchern separat als Adjektive aufgeführt werden. So existiert für die Verben ausdienen oder abdanken neben dem regulär gebildeten (verbalen) Partizip auch ein gleichlautendes, als Adjektiv lexikalisiertes Partizip, das in der Attribution mit Subjektbezug (aufgrund seiner Lexikalisierung) ohne Adaption auskommt:

(59-1) Zwischen rostigen Fahrrädern, einem ausgedienten Segelboot und staubigen Paletten steht das Gefährt, das sie momentan zwölf Stunden täglich beschäftigt.

(59-2) Kann der abgedankte Chef des Weißen Hauses auf die Gnade der späten Erkenntnisse hoffen, so werden die Folgen der Geheimoperationen seinem Nachfolger noch zu schaffen machen.

Auch wenn diese Partizipien diachron eine besondere Entwicklung genommen haben ${ }^{26}$, synchron ist kein Unterschied $\mathrm{zu}$ den Beispielen in (56) feststellbar: Auch diesen Partizipien liegt ein intransitives einstelliges Verb zugrunde, das haben als Auxiliar selegiert, sowohl das formale als auch das semantische Verhältnis von Partizip und Basisverb ist regulär und die Bedeutung des Partizips damit vollständig transparent. Einzig der Bezug auf das Subjekt ist - wie auch bei den oben angeführten Beispielen - nicht regelkonform. Entscheidend für ihre Interpretation ist in diesem Zusammenhang, dass das Subjektargument immer auch thematisch in das Verbalereignis eingebunden ist, indem es zugleich die Spezifizierung als $X$ beinhaltet: $j m d$. dankt $a b$ als Intendant - der abgedankte Intendant. Ganz ähnlich erfolgt die Adaption in den folgenden Beispielen mit Appositionen, auch hier muss diese Art der Spezifizierung für die Interpretation mit herangezogen werden:

26. Etymologische Kenntnisse darüber, ob diese Verben jemals transitiv waren, spielen für die Interpretation keine Rolle. Für ausdienen ist anzumerken, dass es ein defizitäres Flexionsparadigma besitzt (keine Präsens- und Präteritalform). 
(60-1) [...] das war in den Augen seiner Familie Simon auch, ein abgebrochener Maler, ein abgebrochener oder gar nicht erst angefangener Bürgerkriegssoldat in Spanien damals [...]

(60-2) Der nunmehr aufgehörte Kolumnist hat in seinen kleinen alternativen Besinnungsfeuilletons jedenfalls selten ein Thema verfehlt, denn die Themen sind ziemlich egal.

Diese Form der Adaption ist für das Partizip II nicht mehr möglich, wenn durch das Einfügen eines zusätzlichen thematischen Arguments wie in: *ein mit dem Diskutieren aufgehörter Kolumnist beide nicht mehr gleichgesetzt werden können. Erwartungsgemäß gilt diese Bedingung nicht für den regelkonformen Gebrauch mit dem Partizip I: ein (mit dem Diskutieren) aufhörender Kolumnist ist nicht zwangsläufig jemand, der auch gerade aufhört, als Kolumnist tätig zu sein.

Betrachten wir noch weitere Partizipien II, die von den Grammatiken als lexikalisiert eingestuft werden, wenn sie subjektbezogen vorkommen. Sie können uns weiteren Aufschluss darüber geben, unter welchen Bedingungen eine Argumentadaption für attributive Partizipien möglich ist. Der Unterschied zu den bisher in (57) und (58) diskutierten Beispielen (und der Grund für die Lexikalisierung) besteht darin, dass das Basisverb keine lexikalischen Varianten zulässt, sondern ausschließlich transitiv vorkommt. Dementsprechend gibt es für das attributive Partizip II neben dem irregulären Subjektbezug wie in (61-1) und (62-1) - auch die Vorkommen, in denen das direkte Objekt als Bezugsnomen gewählt wird, wie die regelkonform gebildeten Pendants in (61-2) und (62-2) zeigen, vgl.

(61-1) Seit mehr als zwanzig Jahren engagiert sich der gelernte Elektromeister in der IG Metall.

(61-2) Wichtiger als die gelernten Zaubersprüche sind die Freundschaften, die der Zauberlehrling Krabat schließt, schmerzvoller als die Schläge des Meisters ist das Erwachsenwerden.

(62-1) Immer wieder kommt in ihm der studierte Politologe zum Vorschein, der an seinem Manuskript klebt und abstrakte Begriffe und Schachtelsätze mag.

(62-2) Doch seit beide 1983 vor der Militärdiktatur in Argentinien nach Spanien flohen, haben sie ihre studierten Berufe nicht mehr ausüben können. 
Für die nicht regulären Vorkommen in (61-1) und (62-1) gilt wieder, dass das Bezugsnomen nicht nur das Agens des zugrunde liegenden Verbs realisiert, sondern auch auf andere Weise an dem Verbalereignis beteiligt ist: das jeweilige Bezugsnomen Elektromeister bzw. Politologe muss zugleich als Resultat des entsprechenden Verbalereignisses interpretiert werden. Wie bei den Adaptionen unter (60) kann das Partizip in diesen Fällen keine weiteren Argumente zu sich nehmen: ein (*Mathematik) studierter Mann. ${ }^{27}$

Allen bisher betrachteten Verben ist gemeinsam, dass sich ihr attributives Partizip II auf das Subjekt beziehen kann. Ihnen ist auch gemeinsam, dass dieses durch das Verbalereignis affiziert bzw. effiziert ${ }^{28}$ wird. Wenn also das Subjektargument als Bezugsnomen für ein Partizip II auftritt, dann muss es eine Veränderung erfahren, durch die es auch nach Abschluss des Verbalereignisses charakterisierbar bleibt. Je stärker ein Argument in das Verbalereignis involviert ist und einer Veränderung unterliegt, desto enger scheint seine Affinität zum Partizip II zu sein und desto weniger markiert ist eine entsprechende Adaption. Für die Möglichkeit zur Bildung von attributiven Partizipien II spielen folglich nicht allein die syntaktischen, sondern auch die semantischen Eigenschaften des Basisverbs (und seiner Argumente) eine Rolle.

Diese Annahme wird gestützt durch das Verhalten der Partizipien von zwei diesbezüglich interessanten Verbklassen: die der Bewegungsverben und die der inhärent reflexiven Verben. Von Bewegungsverben können beide Partizipien pränominale Attribute bilden, wobei dann sowohl das Partizip I als auch das Partizip II regulär subjektbezogen auftritt. Demzufolge lassen sie auch die mit den Partizipien einhergehende aspektuelle Differenzierung ohne Adaption zu:

\section{ein ins Meer laufendes / gelaufenes Kind}

Mit einem expliziten Richtungsargument wie in (63) unterliegt das Subjekt einer Ortsveränderung, durch die es auch nach Abschluss des Verbalereignisses charakterisierbar bleibt. Doch auch ohne eine direktionale Ergänzung ist eine pränominale Attribution mit dem Partizip II nicht immer ungrammatisch:

27. Die obligatorische Argumentreduktion kann als weiteres Indiz für die Lexikalisierung dieser Partizipien gewertet werden.

28. Ein affiziertes Argument ist von dem Verbalereignis betroffen: Sie liest einen Roman. Ein effiziertes Argument wird durch das Verbalereignis hervorgebracht: Sie schreibt einen Roman. Ein affiziertes bzw. effiziertes Argument trägt typischerweise die thematische Rolle PATIENS und wird nicht als Subjekt, sondern als (direktes) Objekt realisiert. 
(64-1) Denn ein Galopper im Training kostet seinen Besitzer im Jahr zwischen 15000 und 18000 Mark, der Geldgewinnpreis je gelaufenes Pferd betrug jedoch rein rechnerisch im vergangenen Jahr nur 8886 Mark.

(64-2) Toni sprang hoch und wuchtete den Ball Richtung Tor, traf aber nur den Kopf des mit gesprungenen Carlos Marchena.

Der zusätzliche interpretatorische Aufwand besteht hier darin, aus dem Kontext eine alternative Eigenschaft zu ermitteln, die mit der dem Subjekt zugeschriebenen kontrastiert. Diese Alternative kann eine andere Bewegungsart (nicht gesprungen, sondern gelaufen) oder einfach - wie in diesem Fall - die Negation sein. Allein die Tatsache, dass gelaufen oder gesprungen wurde, ist in diesem Kontext relevant und macht das Subjekt nur durch das Partizip II (ohne weitere Modifikatoren) charakterisierbar.

Die andere in diesem Zusammenhang aufschlussreiche Verbklasse ist die der inhärent reflexiven Verben. Das Reflexivpronomen ist in diesem Fall kein thematisches Argument des Verbs, das heißt, inhärent reflexive Verben besitzen mit ihm kein Objekt, das als Bezugsnomen für das Partizip II fungieren könnte. Das Partizip II kann jedoch subjektbezogen vorkommen, wenn das Subjekt entsprechend affiziert ist. ${ }^{29}$ In diesen Fällen ist - wie bei den Bewegungsverben auch - die Aspektopposition regulär realisierbar:
a. die sich verliebenden / verliebten Urlauber
b. die sich erholenden / erholten Urlauber
c. die sich betrinkenden / betrunkenen Urlauber

Der reguläre Subjektbezug ist in diesem Zusammenhang hingegen blockiert, wenn das Verb neben dem Reflexivpronomen ein thematisches Argument im Akkusativ fordert, das dann immer den Vorrang als Bezugsnomen erhält:

$$
\begin{aligned}
& \text { das (sich) angeeignete Wissen / *der (sich) Wissen angeeignete } \\
& \text { Schüler }
\end{aligned}
$$

Bisher haben wir die Bedingungen diskutiert, wann das Partizip II eines intransitiven Verbs (regelhaft oder adaptiv) als pränominales Attribut

29. Für die attributive Verwendung des Partizip II reflexiver Verben sind jedoch noch eine Reihe weiterer Faktoren ausschlaggebend. So scheint v. a. auch die Telizität des Basisverbs eine Rolle zu spielen: das verspätete / *beeilte Mädchen, vgl. Kunze (1997). 
des Subjekts auftreten kann, und zwar dann, wenn keine weiteren Argumente vorhanden sind und/oder das Subjektargument nicht (nur) agentiv in das Verbalereignis involviert ist. Dass die Möglichkeit zur pränominalen Attribution eines Partizips II nicht allein von der Art der syntaktischen Realisierung eines Arguments abhängig ist, lässt sich auch anhand intransitiver Verben zeigen, deren attributive Partizipien II nicht regelkonform vorkommen, obwohl sie objektbezogen sind.

Betrachten wir dazu die zweite Gruppe, vgl. Beispiel (55-2): Hier handelt es sich um Verben, die lediglich über ein implizites Objekt verfügen. Ein implizites Objekt wird syntaktisch nicht realisiert - die Verben sind also intransitiv -, dennoch kann es bei attributiver Verwendung des Partizip II in der Position des Bezugsnomens auftauchen. Die hier notwendige Adaption ist problemlos möglich:

(67-1) Bei dieser Weltbesichtigung hatte der Reporter Scholl-Latour Gefahren zu bestehen, wie wir sie nur aus den geschwindelten Abenteuern Karl Mays kennen.

(67-2) Später reihen sich die frühen Erinnerungsfetzen im Vor- und Rückwärtsgang in die gelogenen Passagen des niemals fertigen Lebensfilms ein.

Mit den Bildungen der dritten Gruppe, vgl. Beispiel (55-3), liegen intransitive Verben vor, die einen lexikalischen oder präpositionalen Kasus regieren. Ihr Partizip II kann ebenfalls als pränominales Attribut auftreten, dementsprechend erscheinen dann Dativobjekte (vgl. (68-1)-(68-3)) oder auch Präpositionalobjekte (vgl. (68-4) und (68-5)) nicht regelkonform in der Position des Bezugsnomens:

(68-1) Nach im Gewerbe umlaufenden, nicht widersprochenen Berichten hat er den Anstalten "Barbarei im kollegialen Zusammenleben" vorgeworfen.

(68-2) Die zumal in der SPD gehuldigte beamtenhafte Vorstellung von der sauer erdienten Ochsentour ist antiquiert.

(68-3) Die geschmeichelte Nachwelt läßt sich gern solche kulturhistorischen Treppenwitze einreden, aber sie würde sich keinen Augenblick getroffen fühlen, wenn heute ein neuer Kandidat erschiene.

(68-4) Nicht zuletzt, weil der assistierte Freitod in einigen Ländern wie den Niederlanden und der Schweiz ohnehin schon teilweise erlaubt ist. 
(68-5) Die Zauberflöte, mit Musik von unserem Kapellmeister Mozart, die mit großen Kosten und vieler Pracht in den Dekorationen gegeben wird, findet den gehofften Beifall nicht, weil der Inhalt und die Sprache des Stücks gar zu schlecht sind.

Kennzeichnend für diese Belege ist, dass sie generell markierter sind als die bisher diskutierten. Sie besitzen eine geringere Akzeptabilität und Häufigkeit, vermutlich weil ein lexikalischer bzw. präpositionaler Kasus immer realisiert sein muss. ${ }^{30}$ Eine Adaption in den Beispielen unter (68) ist möglich, weil die Argumentstruktur der zugrunde liegenden Verben genau ein Objekt bereitstellt, das damit als Bezugsnomen immer eindeutig in seiner semantischen Funktion bzw. in seiner thematischen Rolle identifizierbar ist. Die Form der syntaktischen Realisierung eines Arguments ist dabei nicht ausschlaggebend, so gibt es z. B. auch innerhalb der dativregierenden Verben deutliche Akzeptabilitätsunterschiede $(*$ der geholfene Mann vs. der geschmeichelte Mann), die folglich nicht allein auf die Art des geforderten Kasus zurückzuführen sind.

Auch für die Bildungen aus der zweiten und dritte Gruppe ist wieder ein Muster denkbar, dem sie folgen, nämlich das der transitiven Verben. Wie im regulären Falle zugrunde liegender transitiver Verben werden diesen Bildungen, sofern das entsprechende Bezugsnomen Objektstatus hat, passivisch interpretiert. Besonders deutlich wird die Analogiebildung, wenn sie in direkter Koordination mit dem Partizip II eines transitiven Verbs vorkommt; entsprechend unauffällig kann die jeweilige Abweichung dann sein:

(68-1) Der Preis wird von Tag zu Tag festgelegt; und steht und fällt mit der Menge des angebotenen und nachgefragten Öls.

(69-2) Der seit Jahren gehuldigte und verlorene Sohn fügte sich wieder ein in die Lücke beim 1. FC Köln, die er vor 16 Jahren hinterlassen hatte.

(69-3) Bei der Gelegenheit kann man sich auch das Vertrauen der geschmeichelten oder verletzten Kollegen erschleichen und manche gute Idee klauen.

30. Die prädikative Funktion des Partizips ist (im Gegensatz zur attributiven) oftmals regelkonform zulässig, u. a. weil dann das Argument im geforderten Kasus realisiert werden kann, vgl.

(i) Ihm wäre geholfen, wenn es einen Nachlaß auf die Steuerschuld gäbe, der sich nicht parallel zum Einkommen verändert. 
Diese Daten lassen sich auch in den Kontext des Sprachwandels stellen: Sie reflektieren die generelle Tendenz des Deutschen, lexikalische Kasus abzubauen. Für Verben bedeutet das ihre Transitivierung ${ }^{31}$, die dann entsprechend in der attributiven Verwendung des Partizips II eine Art Vorläufer findet. Welche anderen Faktoren eine objektbezogene Adaption begünstigen oder auch blockieren können, muss hier offen bleiben. Wichtig ist jedoch, dass diese Regelverletzungen - anders als in den Adaptionen, die den Subjektbezug betreffen - nicht dazu dienen, eine aspektuelle Differenzierung zu ermöglichen. Es geht hier allein darum, die prinzipiell frei verfügbare Form des Partizip II intransitiver Verben als pränominales Attribut nutzbar zu machen.

Das Spektrum der dargestellten Adaptionsmöglichkeiten hat gezeigt, dass attributive Partizipien hinsichtlich der argumentstrukturellen Interpretation ihres jeweiligen Bezugsnomens über eine größere Flexibilität verfügen, als ihnen die Grammatiken allgemein zuschreiben. Andererseits kann jedoch nicht einfach die Argumentstruktur des Basisverbs völlig ignoriert und adaptiv eine beliebige semantische Beziehung zwischen attributivem Partizip und Bezugsnomen hergestellt werden. Welche Bildungsvoraussetzungen und Grenzen für die Argumentadaption gelten, soll im Folgenden näher untersucht werden.

\subsection{Grenzen für die Argumentstrukturadaption}

Eine Adaption bezüglich der Argumentstruktur wird prinzipiell dadurch ermöglicht, dass die semantische Beziehung zwischen attributivem Partizip und Bezugsnomen (also die thematische Rolle des Bezugsnomens) nicht nur mit dem Partizip, sondern immer auch mit der Argumentstruktur des zugrunde liegenden Verbs vorgegeben ist - im Gegensatz zur aspektuellen Markierung, die allein in der Bedeutung des Partizips verankert und daher invariabel ist. Eine Adaption hinsichtlich der Argumentstruktur ist folglich dann nicht mehr möglich, wenn bei der Interpretation nicht (mehr) eindeutig rekonstruierbar ist, um welches Argument des zugrunde liegenden Verbs es sich beim gegebenen Bezugsnomen handelt. Für Partizipien aus Basisverben mit nur einem Argument (dem Subjekt) bestehen prinzipiell keine Mehrdeutigkeiten, hier treten beide Partizipien attributiv auf, da sie sich interpretatorisch nur auf das Sub-

31. Besonders gut nachvollziehbar ist dieser syntaktische Wandel am Beispiel des Verbs erinnern. Der für das Objekt geforderte Kasus wechselt von Genitiv über Präpositionalkasus zum Akkusativ: Ich erinnere mich dessen/an das/das - eine attributive Verwendung ist möglich, vgl.

(i) Der kaum noch erinnerte Philosoph Herbert Marcuse hat vor Zeiten diese Entwicklung prophetisch auf den Punkt gebracht. 
jekt beziehen können. Für Partizipien aus Basisverben mit mehreren Argumenten ist dagegen die eindeutige Rekonstruierbarkeit nicht ohne Weiteres gegeben. Mit dieser Voraussetzung lassen sich u. a. folgende Beschränkungen für Adaptionen ableiten:

Für das Partizip II hat in der Interpretation immer das verbnächste Argument als Bezugsnomen den Vorrang. Bei einem transitiven Verb ist es das direkte Objekt; für dessen Partizip II ist damit ein Subjektbezug immer blockiert. Um die Merkmalsbündelung Subjektbezug und Perfektivität zu realisieren, steht als pränominale Attribution nur die komplexere Form mit dem Partizip I des Perfektauxiliars zur Verfügung:

a. *der (das Buch) gelesene Schüler

b. der (das Buch) gelesen habende Schüler

Auch wenn die Argumentstruktur des Basisverbs mehrere Objekte beinhaltet, müssen sich das Bezugsnomen und das verbnächste Argument entsprechen. Für mehrere Objekte gilt damit, dass der strukturelle Kasus immer vor dem lexikalischen Kasus den Vorrang hat. Ein Bezug auf das Subjekt oder ein anderes Objekt ist in diesen Fällen ausgeschlossen:
a. die (dem Spieler) gegebenen Karten / *der (die Karten) ge- gebene Spieler
b. der der Lüge bezichtigte Dieb / *die (den Dieb) bezichtigte Lüge
c. die dem Schüler eingefallene Lösung / *der die Lösung einge- fallene Schüler

Wie wir bereits oben (s. Beispiele unter (68-1)-(68-3)) gesehen haben, ist für ein Argument mit lexikalischem Kasus eine Adaption nicht generell ausgeschlossen, sondern eben nur dann, wenn es mit einem Objekt im strukturellen Kasus konkurriert. Nicht ganz so eindeutig ist diese Hierarchie der Argumente bei Partizipien, deren Basisverben den doppelten Akkusativ regieren, hier scheinen beide Objekte gleichermaßen als Bezugsnomen möglich zu sein:
a. die (die Schüler) abgefragten Vokabeln
b. die (die Vokabeln) abgefragten Schüler

Gibt es nun auch für das (regulär subjektbezogene) Partizip I Adaptionsmöglichkeiten? Unsere Korpusrecherche in der ZEIT nach einem (irregulären) Objektbezug ergab keine Treffer. In Internetforen u. ä. Quellen lassen sich zwar vereinzelt Belege finden, die zwar allesamt als sehr markiert, kaum interpretierbar und oftmals aufgrund der formalen Ähnlichkeit von Partizip I und II eher als Tippfehler einzustufen sind, vgl. 
(73-1) ?Schäden, wie zum Beispiel ein Unfall, der durch vergessende Sicherheitsmaßnahmen entstand, werden von der Versicherung nicht gedeckt. Auch ein vergessener Schlüssel im Auto oder fehlender Sprit im Tank sind nicht im Versicherungsschutz enthalten (...)

http://www.im-auto.de/kfz-versicherung/schutzbrief.html

(04. 07. 2011)

Eine entsprechende Adaption ist hier im Allgemeinen nicht möglich, weil in der Interpretation das Subjekt als Bezugsnomen immer den Vorrang erhält. Besonders deutlich wird das am Beispiel von Basisverben mit mehreren lexikalischen Varianten und Argumentstruktur-Alternation. Auch hier ist immer die Interpretation des Bezugsnomens als Subjekt zwingend, was zur Folge hat, dass die jeweils zugrunde liegenden Verben nur als intransitiviert verstanden werden können: ${ }^{32}$

$$
\begin{aligned}
& \text { a. die kochende Suppe } \\
& \text { b. das schmelzende Eis }
\end{aligned}
$$

(intransitives haben-Verb) (intransitives sein-Verb)

Da fast alle Verben des Deutschen über ein Subjekt verfügen, ergibt sich, dass der argumentstrukturelle Interpretationsspielraum des Partizip I gegenüber dem des Partizip II wesentlich eingeschränkt ist.

Als Testfeld für die hier betrachteten Adaptionsmöglichkeiten und -beschränkungen attributiver Partizipien I können unpersönliche Verben herangezogen werden. Für diese Verben sollte es regelkonform kein attributives Partizip I geben, weil die Argumentstruktur keine Theta-Rolle für ein Subjekt bereitstellt und diese Position syntaktisch durch ein Expletivum gefüllt wird. Auch wenn diese Verben im Deutschen sehr selten sind, lassen sich attributive Vorkommen ihres Partizip I belegen:

(75-1) Steiner beherrscht die hohe Kunst der Auslassung, und er arbeitet mit einer seltsam anmutenden, hapernden Mitteilungslogik.

(75-2) Oft genug schon wurden kriselnde Ehen der Royals aufrechterhalten, um zu demonstrieren, dass die Mitglieder des Königshauses nicht die gleichen Fehler wie Normalsterbliche begehen.

(75-3) Maischberger auf N-TV entspricht solchen Qualitätsmaßstäben mehr als viele menschelnde Quasselrunden.

32. Im Gegensatz zum attributiven Partizip II, für das hier sowohl der Subjekt- als auch der Objektbezug als regelkonforme Interpretation zulässig ist:

(i) das geschmolzene Eis (transitives Verb oder intransitives sein-Verb)

(ii) der informierte Leser (transitives oder reflexives Verb) 
Die hier zugrunde liegenden Verben fordern ein Präpositionalobjekt (es hapert an etwas) oder eine lokale Ergänzung (es menschelt/kriselt in) ${ }^{33}$, und beide können als Bezugsnomen des Partizips auftreten. Damit bestätigt sich unsere Vermutung, dass die eingeschränkten Adaptionsvorkommen für das Partizip I tatsächlich darauf zurückzuführen sind, dass das Subjekt in der Interpretation immer den Vorrang erhält. Wenn das zugrunde liegende Verb nicht über ein thematisches Subjekt verfügt, ist das attributive Partizip I adaptiv auch auf andere Argumente beziehbar.

Stellt das Basisverb keinerlei Argumente bereit und sind demzufolge auch keine thematischen Rollen vorgegeben, wie beispielsweise bei Witterungsverben, so kommen ihre Partizipien meistens in metaphorischen oder resultativen Bildungen vor (regnende Rosen, nass geregnete Gäste). Prinzipiell ist das Partizip dann jedoch frei verfügbar, so dass es sogar auf temporale (seltener auch auf lokale) Adverbiale bezogen sein kann, weil sie eindeutig als solche identifizierbar sind:

(76-1) Wer jetzt an diesem dunkelnden Herbstnachmittag am Bungalow von Eero Aarnio vorbeikommt, muss den Eindruck haben, darin erscheine gerade ein Engel.

(76-2) Denn morgen muss man selbst hinaus in den dunklen, nieselnden Dezember, Kragen hoch und keinen Schirm dabei.

(76-3) Ich versuche meine konfus umherschweifenden Gedanken beim Anblick der dämmernden Stadt nicht einmal zu ordnen.

Unabhängig davon, wie flexibel die semantische Relation zwischen Bezugsnomen und Partizip auch sein mag, der aspektuelle Bedeutungsbeitrag des Partizips bleibt stets invariant. Auch bei unpersönlichen Verben kann das Partizip I nur imperfektiv, das Partizip II nur perfektiv interpretiert werden:

\section{Die vom Himmel regnende / geregnete Blausäure [...]}

Fazit: Argumentadaptionen kommen bei beiden Partizipien nur vor, wenn ein regelhafter Argumentbezug nicht möglich ist. Das Partizip I eines Basisverbs mit thematischem Subjekt steht folglich ebenso wenig für Argumentadaptionen zur Verfügung wie das Partizip II eines transitiven Verbs. Aus diesen Restriktionen erklärt sich, warum Argumentadaptionen beim Partizip I selten auftreten: es gibt im Deutschen nur sehr wenige Verben ohne thematisches Subjekt, die hierfür in Frage kämen.

33. Mit der Einordnung dieser Verben als "unpersönlich" und den Angaben zu ihrer Argumentsstruktur folgen wir den Angaben im Duden (1999, 2001) und Wahrig (2008). 


\section{Zusammenfassung}

Mit dem vorliegenden Beitrag haben wir zwei Adaptionsmechanismen hinsichtlich der Interpretation von attributiven Partizipien vorgestellt: die temporale und die argumentstrukturelle Adaption.

Die temporale Adaption beruht darauf, dass die Partizipien - als infinite Verbalformen - hinsichtlich der grammatischen Kategorie Tempus nicht markiert sind, aber durchaus temporal interpretiert werden, und zwar auch dann, wenn keine explizite Spezifizierung durch ein Zeitadverbiale erfolgt. Temporale Adaption muss als Bedeutungsanreicherung verstanden werden, ausgelöst auf pragmatischer Ebene durch die Maxime der Relevanz; dabei wird das Partizip I im Regelfall als gleichzeitig zur Matrixtopikzeit verstanden, das Partizip II dagegen als vorzeitig. Spezifische Kontexte können diese temporalen Implikaturen jedoch aufheben.

Die argumentstrukturelle Adaption betrifft die Interpretation des Bezugsnomens eines attributiven Partizips. Argumentadaptionen können prinzipiell nur auftreten, wenn der durch das jeweilige Partizip eigentlich vorgegebene Argumentsbezug nicht realisiert werden kann; d. h. im Falle des Partizip II bei Basisverben ohne direktes Objekt, im Falle des Partizip I nur bei Basisverben ohne thematisches Subjekt. Im Gegensatz zur temporalen Interpretation ist diese Form der Adaption keine Anreicherung, sondern eine Uminterpretation von (syntaktischer) Information. Es liegt also eine Regelverletzung vor, die repariert werden muss. Argumentadaptionen treten fast ausschließlich beim Partizip II auf. Adaptionen mit Objektbezug richten sich dabei nach dem Muster der transitiven Verben. Am häufigsten ist eine Argumentadaption bei intransitiven, telischen haben-Verben: hier erlaubt die Wahl zwischen den beiden Partizipien - wie bei den intransitiven sein-Verben - eine Differenzierung zwischen Imperfektivität und Perfektivität. Somit bestätigen auch Argumentadaptionen, dass der wichtigste Bedeutungsbeitrag der Partizipialmorphologie die aspektuelle Markierung eines Verbalereignisses ist.

Eingereicht: 13. August 2010

Überarbeitete Fassung eingereicht: 23. Mai 2011

Sonderforschungsbereich 833
Universität Tübingen
anja.luebbe@uni-tuebingen.de
Deutsches Seminar
Universität Tübingen
irene.rapp@uni-tuebingen.de

\section{Literatur}

Bäuerle, Rainer. 1978. Temporale Deixis, temporale Frage. Zum propositionalen Gehalt deklarativer und interrogativer Sätze (Ergebnisse und Methoden moderner Sprachwissenschaft 5). Tübingen: Narr.

Bech, Gunnar. 1983 [1955/1957]. Studien über das deutsche verbum infinitum. 2. edn. Tübingen: Niemeyer. 
Behaghel, Otto. 1924. Deutsche Syntax. Eine geschichtliche Darstellung. Vol. II. Heidelberg: Carl Winters Universitätsbuchhandlung.

Bresson, Daniel \& Martine Dalmas (eds.). 1994. Partizip und Partizipialgruppen im Deutschen (Eurogermanistik 5). Tübingen: Narr.

Burzio, Luigi. 1981. Intransitive Verbs and Italian Auxiliaries. Cambridge, MA: Massachusettes Institut of Technology (MIT) dissertation.

Dowty, David R. 1979. Word meaning and Montague grammar: the semantics of verbs and times in generative semantics and in Montague's PTQ (Synthese language library 7). Dordrecht: Reidel.

Duden - Das große Wörterbuch der deutschen Sprache in zehn Bänden. 1999. Mannheim: Dudenverlag.

Duden - Deutsches Universalwörterbuch. ${ }^{4}$ 2001. Mannheim: Dudenverlag.

Duden - Richtiges und gutes Deutsch. Wörterbuch der sprachlichen Zweifelsfälle (Der Duden in zwölf Bänden 9). ${ }^{6}$ 2007. Mannheim: Dudenverlag.

Ehrich, Veronika \& Heinz Vater. 1989. Das Perfekt im Dänischen und im Deutschen. In Werner Abraham \& Theo Janssen (eds.), Tempus - Aspekt - Modus. Die lexikalischen und grammatischen Formen in den germanischen Sprachen, 103-132. Tübingen: Niemeyer.

Enç, Mürvet. 1986. Towards a Referential Analysis of Temporal Expressions. Linguistics and Philosophy 9. 405-426.

Faucher, Eugène. 1994. Partizip oder Adjektiv? Partizip oder Infinitiv? Benennungs- und Abgrenzungsfragen. In Daniel Bresson \& Martine Dalmas (eds.), Partizip und Partizipialgruppen im Deutschen, 1-18. Tübingen: Narr.

Fuhrhop, Nanna \& Oliver Teuber. 2000. Das Partizip 1 als adjektivischer Infinitiv. In Andreas Bittner, Dagmar Bittner \& Klaus-Michael Köpcke (eds.), Angemessene Strukturen: Systemorganisation in Phonologie, Morphologie und Syntax, 173-190. Hildesheim: Georg Olms.

Givón, Talmy. 1984. Syntax. A functional-typological introduction. Amsterdam: Benjamins.

Grewendorf, Günther. 1989. Ergativity in German. Foris: Dordrecht.

Grice, H. Paul. 1975. Logic and Conversation. In Peter Cole \& Jerry L. Morgan (eds.), Speech acts (Syntax and Semantics 3), 41-58. New York: Academic Press.

Handwerker, Brigitte. 2007. Das semantische und syntaktische Potenzial von Partizipien im Deutschen: Entdeckungen auf einer Reise durch die poetische Welt des Robert Gernhardt. Estudios Filológicos Alemanes 13. 123-137.

Klein, Wolfgang. 1994. Time in Language (Germanic Linguistics). London: Routledge.

Kunze, Jürgen. 1997. Typen der reflexiven Verbverwendung im Deutschen und ihre Herkunft. Zeitschrift für Sprachwissenschaft 16 (1). 83-180.

Kusumoto, Kiyomi. 2005. On the quantification over times in natural language. Natural Language Semantics 13. 317-357.

Landman, Fred. 1992. The progressive. Natural Language Semantics 1 (1). 1-32.

Lehmann, Christian. 1992. Deutsche Prädikatklassen in typologischer Sicht. In Ludger Hoffmann (ed.), Deutsche Syntax. Ansichten und Aussichten, 155-185. Berlin: de Gruyter.

Lenz, Barbara. 1993. Probleme der Kategorisierung deutscher Partizipien. Zeitschrift für Sprachwissenschaft 12 (1). 39-76.

Maienborn, Claudia. 2007. Das Zustandspassiv: Grammatische Einordnung - Bildungsbeschränkungen - Interpretationsspielraum. Zeitschrift für Germanistische Linguistik 35. $83-115$.

Marillier, Jean-Francois. 1994. Was sind Partizipien? In Daniel Bresson \& Martine Dalmas (eds.), Partizip und Partizipialgruppen im Deutschen, 19-32. Tübingen: Narr.

Musan, Renate. 1997. On the Temporal Interpretation of Noun Phrases. New York: Garland Publishing, Inc. 
Pakkanen-Kilpiä, Kirsi. 2004: Zur Verwendbarkeit des Partizips II als Attribut (Finnische Beiträge zur Germanistik 11). Frankfurt/Main: Peter Lang.

Paul, Hermann. 1920. Deutsche Grammatik. Vol. IV. Halle/Saale: Niemeyer.

Perlmutter, David. 1978. Impersonal Passives and the Unaccusative Hypothesis. Berkeley Linguistics Society (BLS) 4. 157-189.

Poitou, Jacques. 1994. Morphologische Analyse und Kategorisierung der Partizipien. In Daniel Bresson \& Martine Dalmas (eds.), Partizip und Partizipialgruppen im Deutschen, 109-120. Tübingen: Narr.

Quintin, Hervé. 1994. Zur morphosyntaktischen und semantischen Einordnung von deutschen Partizipien und Partizipialsätzen. In Daniel Bresson \& Martine Dalmas (eds.), Partizip und Partizipialgruppen im Deutschen, 91-107. Tübingen: Narr.

Rapp, Irene. 1996. Zustand? Passiv? - Überlegungen zum sogenannten "Zustandspassiv". Zeitschrift für Sprachwissenschaft 15 (2). 231-265.

Rapp, Irene. 1997. Partizipien und semantische Struktur: zu passivischen Konstruktionen mit dem 3. Status (Studien zur deutschen Grammatik 54). Tübingen: Stauffenburg.

Rapp, Irene. 2000. The attributive past participle: structure and temporal interpretation. In Caroline Féry \& Wolfgang Sternefeld (eds.), Audiatur vox sapientiae: a festschrift for Arnim von Stechow (studia grammatica 52), 392-409. Berlin: Akademie Verlag.

Reichenbach, Hans. 1947. Elements of Symbolic Logic. New York: Macmillan \& Co.

Strobel, Sven. 2008. Die Perfektauxiliarselektion des Deutschen. Ein lexikalistischer Ansatz ohne Unakkusativität. Stuttgart: Universität Stuttgart Dissertation.

Struckmeier, Volker. 2007. Attribute im Deutschen. Zu ihren Eigenschaften und ihrer Position im grammatischen System. Berlin: Akademie Verlag.

Toman, Jindřich. 1986. A (Word-) Syntax for Participles. Linguistische Berichte 105. 367408.

Valentin, Paul. 1994. Über Nicht-Partizipien und Partizipien im heutigen Deutsch. In Daniel Bresson \& Martine Dalmas (eds.), Partizip und Partizipialgruppen im Deutschen, 3346. Tübingen: Narr.

Wahrig - Deutsches Wörterbuch. (8. edn. 2008). Gütersloh: Wissen-Media-Verlag.

Weber, Heinrich. 2000. Partizip Präsens und Partizip Perfekt im Deutschen - eine Aspektopposition? In Andrzej Kạtny (ed.), Aspektualität in germanischen und slawischen Sprachen (Seria Filologia Germańska 46), 109-124. Poznań: Wydawnictwo Naukowe UAM

Wiese, Richard. 1986. Schwa and the Structure of Words in German. Linguistics 24. 697724.

Wunderlich, Dieter. 1987. Partizipien im Deutschen. Linguistische Berichte 111. 345-366.

Zifonun, Gisela, Ludger Hoffmann \& Bruno Strecker. 1997. Grammatik der deutschen Sprache. Vol. 3. Berlin: de Gruyter.

Zimmer, Dieter E. 2007. Alles eine Sache des Geschmacks? Von wegen! In DIE ZEIT 31/2007, Feuilleton. http://www.zeit.de/2007/31/Alles_eine_Sache_des_Geschmacks. (04. 07. 2011) 